\title{
Design, Synthesis and Evaluation of Novel Auxin Mimic Herbicides
}

Chi-Linh Do-Thanh, ${ }^{1}$ Jose J. Vargas, ${ }^{2}$ Joseph W.Thomas, ${ }^{2}$ Gregory R. Armel ${ }^{2,3 *}$ and Michael D. Best ${ }^{* 1}$

${ }^{1}$ Department of Chemistry, the University of Tennessee, Knoxville, TN 37996

${ }^{2}$ Department of Plant Sciences, the University of Tennessee, Knoxville, TN 37996

${ }^{3}$ Global Herbicide Development Division, BASF Corporation, Research Triangle Park, NC 27709 


\section{Supporting Information}

Index of Contents:

S2 - S9: Synthetic Procedures

S10: References

S10 - S42: NMR Spectra for Synthetic Compounds

\section{Synthetic Procedures}

\section{General Procedure A: Esterification of Aromatic Carboxylic Acids}

Concentrated sulfuric acid $(1 \mathrm{~mL})$ was added to a solution of the carboxylic acid (10 $\mathrm{mmol})$ in methanol $(20 \mathrm{~mL})$. The reaction mixture was stirred at $65^{\circ} \mathrm{C}$ overnight, after which a saturated aqueous sodium bicarbonate solution was added to adjust the $\mathrm{pH}$ to about 8 . The mixture was then extracted twice with dichloromethane, and the combined organic layers were dried with magnesium sulfate, filtered, and concentrated to give the product.

\section{General Procedure B: Palladium-Catalyzed Coupling of Aryl Bromides with 4-} Chlorophenylboronic Acid

The procedure described by Miyaura, Yanagi, and Suzuki ${ }^{1}$ was modified in the following manner. To a suspension of the aryl bromide $(10 \mathrm{mmol})$, bis(triphenylphosphine)palladium(II) chloride $(0.5 \mathrm{mmol})$, and 4-chlorophenylboronic acid $(15 \mathrm{mmol})$ in methanol $(30 \mathrm{~mL})$ and toluene $(30 \mathrm{~mL})$ was added a $2 \mathrm{M}$ aqueous sodium carbonate solution $(20 \mathrm{mmol})$. The reaction mixture was stirred at $80{ }^{\circ} \mathrm{C}$ overnight and then filtered through Celite. The filtrate was extracted twice with diethyl ether from water, and the combined organic layers were dried over magnesium sulfate, filtered, and concentrated. Column chromatography over silica gel afforded the pure product. Compounds were eluted using varying percentages of ethyl acetate in hexanes as eluant.

\section{General Procedure C: Ester Hydrolysis}

A $2 \mathrm{M}$ aqueous sodium hydroxide solution (15 mmol) was added to a suspension of the ester $(5 \mathrm{mmol})$ in methanol $(50 \mathrm{~mL})$. The reaction mixture was stirred at $65^{\circ} \mathrm{C}$ for $3 \mathrm{~h}$ and then extracted twice with dichloromethane from $1 \mathrm{M}$ hydrochloric acid. The combined organic layers were dried over magnesium sulfate, filtered, and concentrated. When necessary, column chromatography over silica gel afforded the pure product. 


\section{methyl 5-bromo-2-chlorobenzoate (9a)}

This compound was prepared, following General Procedure A, from 5-bromo-2chlorobenzoic acid $(8 \mathrm{a}, 2.09 \mathrm{~g}, 8.89 \mathrm{mmol})$ and was obtained as a white solid $(2.08 \mathrm{~g}$, 94\%).

Characterizations matched those reported in the literature..$^{2,3}$

${ }^{1} \mathrm{H}$ NMR $\left(300 \mathrm{MHz}, \mathrm{CDCl}_{3}\right) \delta 7.96(\mathrm{~d}, J=2.5 \mathrm{~Hz}, 1 \mathrm{H}), 7.53(\mathrm{dd}, J=8.5,2.5 \mathrm{~Hz}, 1 \mathrm{H})$, $7.32(\mathrm{~d}, \mathrm{~J}=8.5 \mathrm{~Hz}, 1 \mathrm{H}), 3.94(\mathrm{~s}, 3 \mathrm{H})$.

methyl 2-chloro-5-(4-chlorophenyl)benzoate (10a)

This compound was prepared, following General Procedure B, from 9a $(1.99 \mathrm{~g}, 7.96$ $\mathrm{mmol}$ ). Column chromatography over silica gel with gradient elution from 5 to $15 \%$ ethyl acetate/hexanes gave the product as a white solid (1.66 g, 74\%).

${ }^{1} \mathrm{H}$ NMR $\left(250 \mathrm{MHz}, \mathrm{CDCl}_{3}\right) \delta 8.00(\mathrm{~d}, \mathrm{~J}=2.2 \mathrm{~Hz}, 1 \mathrm{H}), 7.63-7.36(\mathrm{~m}, 6 \mathrm{H}), 3.96(\mathrm{~s}, 3 \mathrm{H})$.

2-chloro-5-(4-chlorophenyl)benzoic acid (1a)

This compound was prepared, following General Procedure C, from 10a $(1.34 \mathrm{~g}, 4.75$ $\mathrm{mmol}$ ). Column chromatography over silica gel with gradient elution from 5 to $30 \%$ methanol/dichloromethane gave the product as a pale yellow solid $(1.25 \mathrm{~g}, 99 \%)$.

${ }^{1} \mathrm{H}$ NMR $\left(300 \mathrm{MHz}, \mathrm{CD}_{3} \mathrm{OD}\right) \delta 8.05(\mathrm{~d}, \mathrm{~J}=2.4 \mathrm{~Hz}, 1 \mathrm{H}), 7.72(\mathrm{dd}, \mathrm{J}=8.4,2.4 \mathrm{~Hz}, 1 \mathrm{H})$, $7.62(\mathrm{~d}, J=8.8 \mathrm{~Hz}, 2 \mathrm{H}), 7.56(\mathrm{~d}, J=8.4 \mathrm{~Hz}, 1 \mathrm{H}), 7.46(\mathrm{~d}, J=8.8 \mathrm{~Hz}, 2 \mathrm{H})$.

methyl 5-bromo-2-fluorobenzoate (9b)

This compound was prepared, following General Procedure A, from 5-bromo-2fluorobenzoic acid $(\mathbf{8 b}, 2.00 \mathrm{~g}, 9.14 \mathrm{mmol})$ and was obtained as a colorless liquid (1.98 g, 93\%).

Characterizations matched those reported in the literature. ${ }^{4}$

${ }^{1} \mathrm{H} \mathrm{NMR}\left(300 \mathrm{MHz}, \mathrm{CDCl}_{3}\right) \delta 8.06$ (dd, $\mathrm{J}=6.3,2.6 \mathrm{~Hz}, 1 \mathrm{H}$ ), 7.62 (ddd, $J=8.8,4.2,2.6$ $\mathrm{Hz}, 1 \mathrm{H}), 7.05$ (dd, J = 10.1, 8.8 Hz, 1H), 3.94 (s, 3H).

\section{methyl 5-(4-chlorophenyl)-2-fluorobenzoate (10b)}

This compound was prepared, following General Procedure B, from 9b $(1.95 \mathrm{~g}, 8.37$ $\mathrm{mmol}$ ). Column chromatography over silica gel with gradient elution from 5 to $15 \%$ ethyl acetate/hexanes gave the product as a white solid $(1.48 \mathrm{~g}, 67 \%)$.

${ }^{1} \mathrm{H}$ NMR $\left(300 \mathrm{MHz}, \mathrm{CDCl}_{3}\right) \delta 8.12$ (dd, $\mathrm{J}=6.8,2.5 \mathrm{~Hz}, 1 \mathrm{H}$ ), 7.69 (ddd, $\mathrm{J}=8.6,4.5,2.5$ $\mathrm{Hz}, 1 \mathrm{H}), 7.50(\mathrm{~d}, \mathrm{~J}=8.8 \mathrm{~Hz}, 2 \mathrm{H}), 7.42(\mathrm{~d}, \mathrm{~J}=8.8 \mathrm{~Hz}, 2 \mathrm{H}), 7.22(\mathrm{dd}, \mathrm{J}=10.3,8.6 \mathrm{~Hz}$, $1 \mathrm{H}), 3.97(\mathrm{~s}, 3 \mathrm{H})$. 


\section{5-(4-chlorophenyl)-2-fluorobenzoic acid (1b)}

This compound was prepared, following General Procedure C, from $10 \mathrm{~b}(1.47 \mathrm{~g}, 5.54$ $\mathrm{mmol}$ ). Column chromatography over silica gel with gradient elution from 5 to $30 \%$ methanol/dichloromethane gave the product as a white solid $(1.01 \mathrm{~g}, 73 \%)$.

${ }^{1} \mathrm{H}$ NMR $\left(300 \mathrm{MHz}, \mathrm{CD}_{3} \mathrm{OD}\right.$ ) $\delta 8.12$ (dd, $J=6.9,2.5 \mathrm{~Hz}, 1 \mathrm{H}$ ), 7.80 (ddd, $J=8.6,4.5,2.5$ $\mathrm{Hz}, 1 \mathrm{H}$ ), 7.59 (d, J = 8.8 Hz, 2H), 7.44 (d, J = 8.8 Hz, 2H), 7.27 (dd, J = 10.5, 8.6 Hz, $1 \mathrm{H})$.

\section{methyl 5-bromonicotinate (12a)}

This compound was prepared, following General Procedure A, from 5-bromonicotinic acid $(11 \mathrm{a}, 2.03 \mathrm{~g}, 10.04 \mathrm{mmol})$ as a white solid $(1.91 \mathrm{~g}, 88 \%)$.

Characterizations matched those reported in the literature. 5,6

${ }^{1} \mathrm{H}$ NMR $\left(300 \mathrm{MHz}, \mathrm{CDCl}_{3}\right) \delta 9.13(\mathrm{~d}, \mathrm{~J}=1.8 \mathrm{~Hz}, 1 \mathrm{H}), 8.84(\mathrm{~d}, \mathrm{~J}=2.3 \mathrm{~Hz}, 1 \mathrm{H}), 8.44(\mathrm{t}, J$ $=2.1 \mathrm{~Hz}, 1 \mathrm{H}), 3.97(\mathrm{~s}, 3 \mathrm{H})$.

\section{methyl 5-(4-chlorophenyl)nicotinate (13a)}

This compound was prepared, following General Procedure B, from 12a $(1.75 \mathrm{~g}, 8.11$ $\mathrm{mmol}$ ). Column chromatography over silica gel with gradient elution from 10 to $50 \%$ ethyl acetate/hexanes gave the product as a light yellow solid $(1.67 \mathrm{~g}, 83 \%)$.

Characterizations matched those reported in the literature. ${ }^{7}$

${ }^{1} \mathrm{H}$ NMR $\left(250 \mathrm{MHz}, \mathrm{CDCl}_{3}\right) \delta 9.20(\mathrm{~d}, \mathrm{~J}=1.7 \mathrm{~Hz}, 1 \mathrm{H}), 8.98(\mathrm{~d}, \mathrm{~J}=2.2 \mathrm{~Hz}, 1 \mathrm{H}), 8.46(\mathrm{t}, \mathrm{J}$ $=2.2 \mathrm{~Hz}, 1 \mathrm{H}), 7.56(\mathrm{~d}, \mathrm{~J}=8.8 \mathrm{~Hz}, 2 \mathrm{H}), 7.48(\mathrm{~d}, \mathrm{~J}=8.8 \mathrm{~Hz}, 2 \mathrm{H}), 3.99(\mathrm{~s}, 3 \mathrm{H})$.

\section{5-(4-chlorophenyl)nicotinic acid (2a)}

This compound was prepared, following General Procedure C, from 13a (1.63 g, 6.59 $\mathrm{mmol}$ ). Column chromatography over silica gel with gradient elution from 10 to $50 \%$ methanol/dichloromethane gave the product as a white solid $(1.34 \mathrm{~g}, 87 \%)$.

${ }^{1} \mathrm{H}$ NMR $\left(300 \mathrm{MHz}, \mathrm{CD}_{3} \mathrm{OD}\right) \delta 9.10(\mathrm{~s}, 1 \mathrm{H}), 8.97(\mathrm{~s}, 1 \mathrm{H}), 8.58(\mathrm{t}, \mathrm{J}=2.1 \mathrm{~Hz}, 1 \mathrm{H}), 7.71$ $(\mathrm{d}, \mathrm{J}=8.8 \mathrm{~Hz}, 2 \mathrm{H}), 7.53(\mathrm{~d}, \mathrm{~J}=8.8 \mathrm{~Hz}, 2 \mathrm{H})$.

\section{methyl 6-bromopicolinate $(12 b)$}

This compound was prepared, following General Procedure A, from 6-bromopicolinic acid $(\mathbf{1 1 b}, 0.33 \mathrm{~g}, 1.58 \mathrm{mmol})$ as a white solid $(0.33 \mathrm{~g}, 95 \%)$.

Characterizations matched those reported in the literature..$^{8-10}$

${ }^{1} \mathrm{H}$ NMR $\left(300 \mathrm{MHz}, \mathrm{CDCl}_{3}\right) \delta 8.11(\mathrm{dd}, \mathrm{J}=7.3,0.8 \mathrm{~Hz}, 1 \mathrm{H}), 7.81-7.68(\mathrm{~m}, 2 \mathrm{H}), 4.01(\mathrm{~s}$, $3 \mathrm{H})$. 


\section{methyl 6-(4-chlorophenyl)picolinate (13b)}

This compound was prepared, following General Procedure B, from $12 \mathrm{~b}(0.17 \mathrm{~g}, 0.78$ $\mathrm{mmol}$ ). Column chromatography over silica gel with gradient elution from 5 to $20 \%$ ethyl acetate/hexanes gave the product as a white solid $(0.084 \mathrm{~g}, 44 \%)$.

${ }^{1} \mathrm{H}$ NMR $\left(300 \mathrm{MHz}, \mathrm{CDCl}_{3}\right) \delta 8.06(\mathrm{~d}, \mathrm{~J}=7.0 \mathrm{~Hz}, 1 \mathrm{H}), 8.00(\mathrm{~d}, \mathrm{~J}=8.7 \mathrm{~Hz}, 2 \mathrm{H}), 7.93-$ $7.83(\mathrm{~m}, 2 \mathrm{H}), 7.45(\mathrm{~d}, \mathrm{~J}=8.7 \mathrm{~Hz}, 2 \mathrm{H}), 4.02(\mathrm{~s}, 3 \mathrm{H})$.

\section{6-(4-chlorophenyl)picolinic acid (2b)}

This compound was prepared, following General Procedure C, from 13b $(0.084 \mathrm{~g}, 0.34$ $\mathrm{mmol}$ ). Column chromatography over silica gel with gradient elution from 5 to $20 \%$ methanol/dichloromethane gave the product as a white solid $(0.072 \mathrm{~g}, 91 \%)$.

Characterizations matched those reported in the literature. ${ }^{11}$

${ }^{1} \mathrm{H}$ NMR $\left(300 \mathrm{MHz}, \mathrm{CD}_{3} \mathrm{OD}\right) \delta 8.16-7.91(\mathrm{~m}, 5 \mathrm{H}), 7.45(\mathrm{~d}, \mathrm{~J}=7.9 \mathrm{~Hz}, 2 \mathrm{H})$.

methyl 5-bromo-6-chloronicotinate (15a)

This compound was prepared according to a literature procedure. ${ }^{12}$ A solution of 5bromo-6-chloronicotinic acid $(14 \mathrm{a}, 0.16 \mathrm{~g}, 0.67 \mathrm{mmol})$ in thionyl chloride $(5 \mathrm{~mL})$ was stirred at $70{ }^{\circ} \mathrm{C}$ for $1 \mathrm{~h}$ and then concentrated by rotary evaporation. Benzene $(10 \mathrm{~mL})$ and anhydrous methanol $(5 \mathrm{~mL})$ were added to the residue, followed by stirring at $65^{\circ} \mathrm{C}$ for $1 \mathrm{~h}$. The reaction mixture was then concentrated and extracted with dichloromethane $(2 \times 20 \mathrm{~mL})$ from saturated aqueous sodium bicarbonate $(20 \mathrm{~mL})$. The combined organic layers were dried over magnesium sulfate, filtered, and concentrated to give the product as a light yellow solid $(0.16 \mathrm{~g}, 98 \%)$.

Characterizations matched those reported in the literature. ${ }^{12}$

${ }^{1} \mathrm{H} \mathrm{NMR}\left(300 \mathrm{MHz}, \mathrm{CDCl}_{3}\right) \delta 8.92(\mathrm{~d}, \mathrm{~J}=2.0 \mathrm{~Hz}, 1 \mathrm{H}), 8.52(\mathrm{~d}, \mathrm{~J}=2.0 \mathrm{~Hz}, 1 \mathrm{H}), 3.98(\mathrm{~s}$, $3 \mathrm{H})$.

methyl 6-chloro-5-(4-chlorophenyl)nicotinate (16a)

This compound was prepared, following General Procedure B, from $15 \mathrm{a}(0.083 \mathrm{~g}, 0.32$ $\mathrm{mmol}$ ). Column chromatography over silica gel with gradient elution from 10 to $30 \%$ ethyl acetate/hexanes gave the product as a white solid $(0.027 \mathrm{~g}, 29 \%)$.

${ }^{1} \mathrm{H}$ NMR $\left(300 \mathrm{MHz}, \mathrm{CDCl}_{3}\right) \delta 8.99(\mathrm{~d}, \mathrm{~J}=2.3 \mathrm{~Hz}, 1 \mathrm{H}), 8.25(\mathrm{~d}, \mathrm{~J}=2.3 \mathrm{~Hz}, 1 \mathrm{H}), 7.47$ (d, $\mathrm{J}=8.8 \mathrm{~Hz}, 2 \mathrm{H}), 7.41(\mathrm{~d}, \mathrm{~J}=8.8 \mathrm{~Hz}, 2 \mathrm{H}), 3.98(\mathrm{~s}, 3 \mathrm{H})$.

\section{6-chloro-5-(4-chlorophenyl)nicotinic acid (3a)}

This compound was prepared, following General Procedure C, from $16 a(0.061 \mathrm{~g}, 0.22$ $\mathrm{mmol}$ ). Column chromatography over silica gel with gradient elution from 3 to $20 \%$ methanol/dichloromethane gave the product as a white solid $(0.049 \mathrm{~g}, 85 \%)$.

${ }^{1} \mathrm{H}$ NMR $\left(300 \mathrm{MHz}, \mathrm{CD}_{3} \mathrm{OD}\right) \delta 8.88(\mathrm{~s}, 1 \mathrm{H}), 8.28(\mathrm{~s}, 1 \mathrm{H}), 7.49-7.39(\mathrm{~m}, 4 \mathrm{H})$. 


\section{methyl 5-bromo-2-chloronicotinate (15b)}

This compound was prepared, in the same manner as $15 a$ described above, from 5bromo-2-chloronicotinic acid $(0.20 \mathrm{~g}, 0.84 \mathrm{mmol})$ as a light yellow solid $(0.20 \mathrm{~g}, 95 \%)$.

Characterizations matched those reported in the literature. ${ }^{12}$

${ }^{1} \mathrm{H}$ NMR $\left(300 \mathrm{MHz}, \mathrm{CDCl}_{3}\right) \delta 8.58(\mathrm{~d}, J=2.5 \mathrm{~Hz}, 1 \mathrm{H}), 8.30(\mathrm{~d}, J=2.5 \mathrm{~Hz}, 1 \mathrm{H}), 3.98(\mathrm{~s}$, $3 \mathrm{H})$.

\section{methyl 2-chloro-5-(4-chlorophenyl)nicotinate (16b)}

This compound was prepared, following General Procedure B, from $15 \mathrm{~b}(0.097 \mathrm{~g}, 0.39$ $\mathrm{mmol}$ ). Column chromatography over silica gel with gradient elution from 10 to $30 \%$ ethyl acetate/hexanes gave the product as a white solid $(0.031 \mathrm{~g}, 28 \%)$.

${ }^{1} \mathrm{H}$ NMR $\left(300 \mathrm{MHz}, \mathrm{CDCl}_{3}\right) \delta 8.69(\mathrm{~d}, \mathrm{~J}=2.6 \mathrm{~Hz}, 1 \mathrm{H}), 8.32(\mathrm{~d}, \mathrm{~J}=2.6 \mathrm{~Hz}, 1 \mathrm{H}), 7.53(\mathrm{~d}$, $\mathrm{J}=8.9 \mathrm{~Hz}, 2 \mathrm{H}), 7.48(\mathrm{~d}, \mathrm{~J}=8.9 \mathrm{~Hz}, 2 \mathrm{H}), 4.00(\mathrm{~s}, 3 \mathrm{H})$.

\section{2-chloro-5-(4-chlorophenyl)nicotinic acid (3b)}

This compound was prepared, following General Procedure C, from $16 \mathrm{~b}(0.11 \mathrm{~g}, 0.39$ $\mathrm{mmol}$ ). Column chromatography over silica gel with gradient elution from 5 to $15 \%$ methanol/dichloromethane gave the product as a white solid $(0.074 \mathrm{~g}, 70 \%)$.

${ }^{1} \mathrm{H}$ NMR $\left(300 \mathrm{MHz}, \mathrm{CD}_{3} \mathrm{OD}\right) \delta 8.63(\mathrm{~s}, 1 \mathrm{H}), 8.31(\mathrm{~s}, 1 \mathrm{H}), 7.65(\mathrm{~d}, \mathrm{~J}=8.2 \mathrm{~Hz}, 2 \mathrm{H}), 7.48$ $(\mathrm{d}, \mathrm{J}=8.2 \mathrm{~Hz}, 2 \mathrm{H})$.

\section{methyl 3-bromo-5-hydroxybenzoate (18)}

This compound was prepared, following General Procedure A, from 3-bromo-5hydroxybenzoic acid $(17,0.10 \mathrm{~g}, 0.45 \mathrm{mmol})$ as a light yellow solid $(0.10 \mathrm{~g}, 100 \%)$.

Characterizations matched those reported in the literature. ${ }^{13,14}$

${ }^{1} \mathrm{H}$ NMR $\left(300 \mathrm{MHz}, \mathrm{CDCl}_{3}\right) \delta 7.74(\mathrm{t}, \mathrm{J}=1.5 \mathrm{~Hz}, 1 \mathrm{H}), 7.54(\mathrm{dd}, \mathrm{J}=2.4,1.5 \mathrm{~Hz}, 1 \mathrm{H})$, $7.25(\mathrm{dd}, \mathrm{J}=2.4,1.8 \mathrm{~Hz}, 1 \mathrm{H}), 6.07(\mathrm{bs}, 1 \mathrm{H}), 3.93(\mathrm{~s}, 3 \mathrm{H})$.

\section{methyl 3-bromo-5-(4-methoxybenzyloxy)benzoate (19)}

To a solution of $18(0.10 \mathrm{~g}, 0.44 \mathrm{mmol})$ and potassium carbonate $(0.090 \mathrm{~g}, 0.65 \mathrm{mmol})$ in $N, N$-dimethylformamide $(10 \mathrm{~mL})$ was added 4-methoxybenzyl chloride $(0.092 \mathrm{~mL}$, $0.65 \mathrm{mmol}$ ) dropwise. The reaction mixture was stirred at $100^{\circ} \mathrm{C}$ overnight and then extracted with ethyl acetate $(2 \times 30 \mathrm{~mL})$ from water $(30 \mathrm{~mL})$. The combined organic layers were dried over magnesium sulfate, filtered, and concentrated. Column chromatography over silica gel with gradient elution from 3 to $15 \%$ ethyl acetate/hexanes gave the product as a colorless oil $(0.14 \mathrm{~g}, 94 \%)$.

${ }^{1} \mathrm{H}$ NMR $\left(300 \mathrm{MHz}, \mathrm{CDCl}_{3}\right) \delta 7.77-7.73(\mathrm{~m}, 1 \mathrm{H}), 7.56(\mathrm{dd}, \mathrm{J}=2.4,1.3 \mathrm{~Hz}, 1 \mathrm{H}), 7.33(\mathrm{~d}$, $\mathrm{J}=8.7 \mathrm{~Hz}, 2 \mathrm{H}), 7.29(\mathrm{dd}, \mathrm{J}=2.4,1.8 \mathrm{~Hz}, 1 \mathrm{H}), 6.91(\mathrm{~d}, \mathrm{~J}=8.7 \mathrm{~Hz}, 2 \mathrm{H}), 4.98(\mathrm{~s}, 2 \mathrm{H})$, $3.89(\mathrm{~s}, 3 \mathrm{H}), 3.80(\mathrm{~s}, 3 \mathrm{H})$. 


\section{methyl 3-(4-chlorophenyl)-5-(4-methoxybenzyloxy)benzoate (20)}

This compound was prepared, following General Procedure B, from $19(2.71 \mathrm{~g}, 7.72$ $\mathrm{mmol}$ ). Column chromatography over silica gel with gradient elution from 5 to $25 \%$ ethyl acetate/hexanes gave the product as a dark yellow solid $(2.17 \mathrm{~g}, 74 \%)$.

${ }^{1} \mathrm{H}$ NMR $\left(300 \mathrm{MHz}, \mathrm{CDCl}_{3}\right) \delta 7.83(\mathrm{t}, \mathrm{J}=1.5 \mathrm{~Hz}, 1 \mathrm{H}), 7.62(\mathrm{dd}, \mathrm{J}=2.5,1.5 \mathrm{~Hz}, 1 \mathrm{H})$, $7.49(\mathrm{~d}, \mathrm{~J}=8.7 \mathrm{~Hz}, 2 \mathrm{H}), 7.41-7.34(\mathrm{~m}, 4 \mathrm{H}), 7.32(\mathrm{dd}, \mathrm{J}=2.5,1.7 \mathrm{~Hz}, 1 \mathrm{H}), 6.92(\mathrm{~d}, \mathrm{~J}=$ $8.7 \mathrm{~Hz}, 2 \mathrm{H}), 5.04(\mathrm{~s}, 2 \mathrm{H}), 3.93(\mathrm{~s}, 3 \mathrm{H}), 3.80(\mathrm{~s}, 3 \mathrm{H})$.

\section{methyl 3-(4-chlorophenyl)-5-hydroxybenzoate (21)}

Trifluoroacetic acid $(5 \mathrm{~mL})$ was added dropwise to a solution of $20(0.12 \mathrm{~g}, 0.30 \mathrm{mmol})$ in dichloromethane $(5 \mathrm{~mL})$ at rt. The reaction mixture was stirred at rt for $3 \mathrm{~h}$, followed by extraction with dichloromethane $(2 \times 20 \mathrm{~mL})$ from water $(20 \mathrm{~mL})$. The combined organic layers were dried over magnesium sulfate, filtered, and concentrated. Column chromatography over silica gel with gradient elution from 20 to $40 \%$ ethyl acetate/hexanes yielded the product as a white solid $(0.078 \mathrm{~g}, 98 \%)$.

${ }^{1} \mathrm{H}$ NMR $\left(300 \mathrm{MHz}, 1: 1 \mathrm{CDCl}_{3}: \mathrm{CD}_{3} \mathrm{OD}\right) \delta 7.72(\mathrm{t}, \mathrm{J}=1.6 \mathrm{~Hz}, 1 \mathrm{H}), 7.54(\mathrm{~d}, \mathrm{~J}=8.7 \mathrm{~Hz}$, $2 \mathrm{H}), 7.45(\mathrm{dd}, \mathrm{J}=2.4,1.4 \mathrm{~Hz}, 1 \mathrm{H}), 7.41(\mathrm{~d}, \mathrm{~J}=8.7 \mathrm{~Hz}, 2 \mathrm{H}), 7.24(\mathrm{dd}, \mathrm{J}=2.4,1.7 \mathrm{~Hz}$, $1 \mathrm{H}), 3.93(\mathrm{~s}, 3 \mathrm{H})$.

\section{3-(4-chlorophenyl)-5-hydroxybenzoic acid (4)}

This compound was prepared, following General Procedure C, from $21(1.12 \mathrm{~g}, 4.28$ $\mathrm{mmol}$ ). Column chromatography over silica gel with gradient elution from 3 to $25 \%$ methanol/dichloromethane gave the product as a white solid $(0.83 \mathrm{~g}, 78 \%)$.

${ }^{1} \mathrm{H}$ NMR $\left(300 \mathrm{MHz}, \mathrm{CD}_{3} \mathrm{OD}\right) \delta 7.72(\mathrm{t}, \mathrm{J}=1.5 \mathrm{~Hz}, 1 \mathrm{H}), 7.55(\mathrm{~d}, \mathrm{~J}=8.7 \mathrm{~Hz}, 2 \mathrm{H}), 7.44$ $(\mathrm{dd}, J=2.4,1.4 \mathrm{~Hz}, 1 \mathrm{H}), 7.40(\mathrm{~d}, J=8.7 \mathrm{~Hz}, 2 \mathrm{H}), 7.21(\mathrm{dd}, J=2.4,1.7 \mathrm{~Hz}, 1 \mathrm{H})$.

methyl 5-bromo-2-chloro-3-nitrobenzoate (23)

5-bromo-2-chlorobenzoic acid $(22,3.01 \mathrm{~g}, 12.77 \mathrm{mmol})$ was added to concentrated sulfuric acid $(29 \mathrm{~mL})$ at $0{ }^{\circ} \mathrm{C}$. A cooled mixture of concentrated sulfuric acid $(1 \mathrm{~mL})$ and concentrated nitric acid $(1 \mathrm{~mL})$ was added dropwise, and the reaction mixture was stirred at rt overnight. The reaction crude was then poured into ice, and the resulting precipitate was filtered and washed with cold water. The precipitate was extracted with ethyl acetate $(2 \times 50 \mathrm{~mL})$ from water $(50 \mathrm{~mL})$, and the combined organic layers were dried over magnesium sulfate, filtered, and concentrated to give a mixture of mostly 5bromo-2-chloro-3-nitrobenzoic acid and some 3-bromo-6-chloro-2-nitrobenzoic acid. Methanol $(20 \mathrm{~mL})$ and concentrated sulfuric acid $(1 \mathrm{~mL})$ were added to the mixture which was then stirred at $65^{\circ} \mathrm{C}$ overnight. A saturated aqueous sodium bicarbonate solution $(15 \mathrm{~mL})$ was then added to adjust the $\mathrm{pH}$ to about 8 , and the mixture was extracted with dichloromethane $(2 \times 30 \mathrm{~mL})$. The combined organic layers were dried over magnesium sulfate, filtered, and concentrated. Column chromatography over silica gel with gradient elution from 10 to $20 \%$ ethyl acetate/hexanes then yielded the pure product as a light yellow solid (1.17 g, 31\% over 2 steps). 
${ }^{1} \mathrm{H}$ NMR $\left(300 \mathrm{MHz}, \mathrm{CDCl}_{3}\right) \delta 8.09(\mathrm{~d}, J=2.4 \mathrm{~Hz}, 1 \mathrm{H}), 7.97(\mathrm{~d}, J=2.3 \mathrm{~Hz}, 1 \mathrm{H}), 3.99(\mathrm{~s}$, $3 \mathrm{H})$.

\section{methyl 3-amino-5-bromo-2-chlorobenzoate (24)}

Iron powder $(0.48 \mathrm{~g}, 8.55 \mathrm{mmol})$ and saturated aqueous ammonium chloride $(1.22 \mathrm{~mL})$ were added to a solution of $23(0.50 \mathrm{~g}, 1.71 \mathrm{mmol})$ in ethanol $(20 \mathrm{~mL})$. The reaction mixture was stirred at $80{ }^{\circ} \mathrm{C}$ for $2 \mathrm{~h}$ and then filtered through Celite. The filtrate was concentrated, and the residue was extracted with ethyl acetate $(2 \times 30 \mathrm{~mL})$ from saturated aqueous sodium bicarbonate $(30 \mathrm{~mL})$. The combined organic layers were dried over magnesium sulfate, filtered, and concentrated. Column chromatography over silica gel with gradient elution from 5 to $20 \%$ ethyl acetate/hexanes gave the product as a white solid $(0.38 \mathrm{~g}, 84 \%)$.

${ }^{1} \mathrm{H}$ NMR $\left(300 \mathrm{MHz}, \mathrm{CDCl}_{3}\right) \delta 7.28(\mathrm{~d}, \mathrm{~J}=2.2 \mathrm{~Hz}, 1 \mathrm{H}), 7.03(\mathrm{~d}, \mathrm{~J}=2.3 \mathrm{~Hz}, 1 \mathrm{H}), 4.32(\mathrm{bs}$, $2 \mathrm{H}), 3.91$ (s, 3H).

methyl 3-amino-2-chloro-5-(4-chlorophenyl)benzoate (25)

This compound was prepared, following General Procedure B, from $24(0.26 \mathrm{~g}, 0.97$ $\mathrm{mmol}$ ). Column chromatography over silica gel with gradient elution from 5 to $20 \%$ ethyl acetate/hexanes gave the product as a light yellow solid $(0.17 \mathrm{~g}, 58 \%)$.

${ }^{1} \mathrm{H}$ NMR $\left(300 \mathrm{MHz}, \mathrm{CDCl}_{3}\right) \delta 7.41(\mathrm{~d}, \mathrm{~J}=8.8 \mathrm{~Hz}, 2 \mathrm{H}), 7.35(\mathrm{~d}, \mathrm{~J}=8.8 \mathrm{~Hz}, 2 \mathrm{H}), 7.31$ (d, $\mathrm{J}=2.2 \mathrm{~Hz}, 1 \mathrm{H}), 7.01(\mathrm{~d}, \mathrm{~J}=2.2 \mathrm{~Hz}, 1 \mathrm{H}), 4.36(\mathrm{bs}, 2 \mathrm{H}), 3.93(\mathrm{~s}, 3 \mathrm{H})$.

\section{3-amino-2-chloro-5-(4-chlorophenyl)benzoic acid (5)}

This compound was prepared, following General Procedure C, from $25(0.25 \mathrm{~g}, 0.83$ $\mathrm{mmol}$ ) as a tan solid $(0.21 \mathrm{~g}, 90 \%)$.

${ }^{1} \mathrm{H}$ NMR $\left(300 \mathrm{MHz}, \mathrm{CD}_{3} \mathrm{OD}\right) \delta 7.56(\mathrm{~d}, \mathrm{~J}=8.8 \mathrm{~Hz}, 2 \mathrm{H}), 7.43(\mathrm{~d}, \mathrm{~J}=8.8 \mathrm{~Hz}, 2 \mathrm{H}), 7.26(\mathrm{~d}$, $\mathrm{J}=2.2 \mathrm{~Hz}, 1 \mathrm{H}), 7.19(\mathrm{~d}, \mathrm{~J}=2.2 \mathrm{~Hz}, 1 \mathrm{H})$.

\section{methyl 5-bromoindole-2-carboxylate (27)}

This compound was prepared, following General Procedure A, from 5-bromoindole-2carboxylic acid $(26,0.32 \mathrm{~g}, 1.35 \mathrm{mmol})$ as a light yellow solid $(0.33 \mathrm{~g}, 96 \%)$.

Characterizations matched those reported in the literature. ${ }^{15,16}$

${ }^{1} \mathrm{H}$ NMR $\left(300 \mathrm{MHz}, \mathrm{CDCl}_{3}\right) \delta 8.99(\mathrm{bs}, 1 \mathrm{H}), 7.85-7.82(\mathrm{~m}, 1 \mathrm{H}), 7.41$ (dd, $\mathrm{J}=8.8,1.9 \mathrm{~Hz}$, $1 \mathrm{H}), 7.31(\mathrm{~d}, \mathrm{~J}=8.8 \mathrm{~Hz}, 1 \mathrm{H}), 7.16-7.13(\mathrm{~m}, 1 \mathrm{H}), 3.96(\mathrm{~s}, 3 \mathrm{H})$.

methyl 5-(4-chlorophenyl)indole-2-carboxylate (28)

This compound was prepared, following General Procedure B, from 27 (0.33 g, 1.29 $\mathrm{mmol}$ ). Column chromatography over silica gel with gradient elution from 5 to $25 \%$ ethyl acetate/hexanes gave the product as a white solid $(0.15 \mathrm{~g}, 40 \%)$. 
${ }^{1} \mathrm{H}$ NMR $\left(300 \mathrm{MHz}, \mathrm{CDCl}_{3}\right) \delta 8.99$ (bs, $\left.1 \mathrm{H}\right), 7.85(\mathrm{~s}, 1 \mathrm{H}), 7.59-7.46(\mathrm{~m}, 4 \mathrm{H}), 7.41(\mathrm{~d}, \mathrm{~J}=$ $8.6 \mathrm{~Hz}, 2 \mathrm{H}), 7.27-7.26(\mathrm{~m}, 1 \mathrm{H}), 3.97(\mathrm{~s}, 3 \mathrm{H})$.

\section{5-(4-chlorophenyl)indole-2-carboxylic acid (6)}

This compound was prepared, following General Procedure C, from $28(0.065 \mathrm{~g}, 0.23$ $\mathrm{mmol}$ ). Column chromatography over silica gel with gradient elution from 3 to $30 \%$ methanol/dichloromethane gave the product as a light yellow solid $(0.062 \mathrm{~g}, 100 \%)$.

${ }^{1} \mathrm{H}$ NMR $\left(300 \mathrm{MHz}, \mathrm{CD}_{3} \mathrm{OD}\right) \delta 7.83(\mathrm{~s}, 1 \mathrm{H}), 7.59(\mathrm{~d}, \mathrm{~J}=8.4 \mathrm{~Hz}, 2 \mathrm{H}), 7.50(\mathrm{~s}, 2 \mathrm{H}), 7.39$ $(\mathrm{d}, \mathrm{J}=8.4 \mathrm{~Hz}, 2 \mathrm{H}), 7.18(\mathrm{~s}, 1 \mathrm{H})$.

\section{methyl 2-(5-bromoindol-3-yl)acetate (30)}

This compound was prepared according to a known procedure. ${ }^{17}$ A solution of chlorotrimethylsilane $(1 \mathrm{~mL})$ in anhydrous methanol $(10 \mathrm{~mL})$ was cooled to $-20{ }^{\circ} \mathrm{C}$ and then added to a solution of 5-bromoindole-3-acetic acid $(29,0.31 \mathrm{~g}, 1.20 \mathrm{mmol})$ in anhydrous methanol $(10 \mathrm{~mL})$. The reaction mixture was stirred at rt overnight and then concentrated by rotary evaporation. The residue was dissolved with dichloromethane, filtered through a pad of silica gel, and washed with $50 \%$ ethyl acetate/hexanes $(50 \mathrm{~mL})$. The filtrate was then concentrated to provide the product as a light brown solid $(0.29 \mathrm{~g}$, $90 \%)$.

Characterizations matched those reported in the literature. ${ }^{17,18}$

${ }^{1} \mathrm{H}$ NMR $\left(300 \mathrm{MHz}, \mathrm{CDCl}_{3}\right) \delta 8.30$ (bs, $\left.1 \mathrm{H}\right), 7.70$ (d, $\left.\mathrm{J}=1.9 \mathrm{~Hz}, 1 \mathrm{H}\right), 7.23$ (dd, $J=8.6$, $1.9 \mathrm{~Hz}, 1 \mathrm{H}), 7.11(\mathrm{~d}, \mathrm{~J}=8.6 \mathrm{~Hz}, 1 \mathrm{H}), 7.02(\mathrm{~d}, \mathrm{~J}=2.4 \mathrm{~Hz}, 1 \mathrm{H}), 3.72-3.71(\mathrm{~m}, 5 \mathrm{H})$.

methyl 2-[5-(4-chlorophenyl)indol-3-yl]acetate (31)

This compound was prepared, following General Procedure B, from $30(0.25 \mathrm{~g}, 0.93$ $\mathrm{mmol}$ ). Column chromatography over silica gel with gradient elution from 20 to $40 \%$ ethyl acetate/hexanes gave the product as a light yellow oil $(0.17 \mathrm{~g}, 60 \%)$.

${ }^{1} \mathrm{H}$ NMR $\left(300 \mathrm{MHz}, \mathrm{CDCl}_{3}\right) \delta 8.19$ (bs, 1H), $7.76(\mathrm{~s}, 1 \mathrm{H}), 7.56(\mathrm{~d}, \mathrm{~J}=8.6 \mathrm{~Hz}, 2 \mathrm{H}), 7.43-$ $7.35(\mathrm{~m}, 4 \mathrm{H}), 7.17(\mathrm{~d}, \mathrm{~J}=2.4 \mathrm{~Hz}, 1 \mathrm{H}), 3.81(\mathrm{~s}, 2 \mathrm{H}), 3.71(\mathrm{~s}, 3 \mathrm{H})$.

\section{2-[5-(4-chlorophenyl)indol-3-yl]acetic acid (7)}

This compound was prepared, following General Procedure C, from $31(0.17 \mathrm{~g}, 0.57$ $\mathrm{mmol}$ ). Column chromatography over silica gel with gradient elution from 5 to $20 \%$ methanol/dichloromethane gave the product as a white solid $(0.072 \mathrm{~g}, 45 \%)$.

Characterizations matched those reported in the literature. ${ }^{19}$

${ }^{1} \mathrm{H}$ NMR (300 MHz, $\left.\mathrm{CD}_{3} \mathrm{OD}\right) \delta 10.42$ (bs, $\left.1 \mathrm{H}\right), 7.77-7.74(\mathrm{~m}, 1 \mathrm{H}), 7.57$ (d, J = 8.3 Hz, $2 \mathrm{H}), 7.42-7.31(\mathrm{~m}, 4 \mathrm{H}), 7.19(\mathrm{~s}, 1 \mathrm{H}), 3.76(\mathrm{~s}, 2 \mathrm{H})$. 


\section{References Cited}

(1) Miyaura, N.; Yanagi, T.; Suzuki, A., The Palladium-Catalyzed Cross-Coupling Reaction of Phenylboronic Acid with Haloarenes in the Presence of Bases. Synth. Commun. 1981, 11, 513-519.

(2) Patrick, D. A.; Bakunov, S. A.; Bakunova, S. M.; Kumar, E. V. K. S.; Lombardy, R. J.; Jones, S. K.; Bridges, A. S.; Zhirnov, O.; Hall, J. E.; Wenzler, T.; Brun, R.; Tidwell, R. R., Synthesis and in Vitro Antiprotozoal Activities of Dicationic 3,5-Diphenylisoxazoles. J. Med. Chem. 2007, 50, 2468-2485.

(3) Lim, S.; Harris, K. J.; Stefany, D.; Gardner, C. J.; Cao, B.; Boffey, R.; Gillespy, T. A.; Aguiar, J. C.; Hunt, H. J.; Dechaux, E. A. 2,6-Substituted-4-Monosubstituted Amino-Pyrimidine as Prostaglandin D2 Receptor Antagonists. World Patent WO/2006/044732, Apr 27, 2006.

(4) Agejas-Chicharro, F. J.; Dressman, B. A.; Sonia, G. S.; Henry, S. S.; Martinez Perez, J. A.; Massey, S. M.; Monn, J. A.; Zia-Ebrahimi, M. S. Pyridyl Derivatives and Their Use as mGlu5 Receptor Antagonists. World Patent WO/2005/094822, Oct 13, 2005.

(5) Thompson, W. J.; Gaudino, J., A general synthesis of 5-arylnicotinates. J. Org. Chem. 1984, 49, 5237-5243.

(6) Potter, B. V. L.; Dowden, J.; Galione, A.; Guse, A. H.; Flügel, A. Therapeutics Comprising Pyridinium Derivatives. World Patent WO/2007/132179, Nov 22, 2007.

(7) Bossenmaier, B.; Friess, T.; Juchem, R.; Kling, L.; Kolm, I.; Krell, H.-W.; Von Hirschheydt, T.; Voss, E. Phenylpyridine Derivatives. U.S. Patent 7429605, Sep 30, 2008.

(8) Kiran, Y. B.; Ikeda, R.; Sakai, N.; Konakahara, T., Single-Step Conversion of Electron-Deficient Aldehydes into the Corresponding Esters in Aqueous Alcohols in the Presence of lodine and Sodium Nitrite. Synthesis 2010, 2010, 276-282.

(9) Collantes, E. M.; Schwarz, J. B. Pyridinyl Amides for the Treatment of CNS and Metabolic Disorders. World Patent WO/2009/098576, Aug 13, 2009.

(10) Giblin, G. M. P.; Hall, A.; Hurst, D. N.; Kilford, I. R.; Lewell, X. Q.; Naylor, A.; Novelli, R. (2-((2Alkoxy)-phenyl)-cyclopent-1-enyl) Aromatic Carbo and Heterocyclic Acid and Derivatives. World Patent WO/2003/084917, Oct 16, 2003.

(11) Oxford, A. W.; Davis, R. J.; Coleman, R. A.; Clark, K. L.; Clark, D. E.; Harris, N. V.; Fenton, G.; Hynd, G.; Stuttle, K. A. J.; Sutton, J. M.; Ashton, M. R.; Boyd, E. A.; Brunton, S. A. EP2 Receptor Agonists. World Patent WO/2005/080367, Sep 1, 2005.

(12) Setliff, F. L.; Huie, W. R., Some Methyl 2,5-Dihalonicotinates and 5,6-Dihalonicotinates. J. Chem. Eng. Data 1981, 26, 332-333.

(13) Khan, F. A.; Choudhury, S., An Efficient Synthesis of Substituted meta-Halophenols and Their Methyl Ethers: Insight into the Reaction Mechanism. Eur. J. Org. Chem. 2010, 2010, 2954-2970.

(14) Lee, Y.; Kelly, M. J., Solid-phase synthesis of phenols and pyridinones via arylboronation/oxidation protocol using aryl bromides. Tetrahedron Lett. 2006, 47, 4897-4901.

(15) Tullberg, E.; Schacher, F.; Peters, D.; Frejd, T., Solvent-Free Heck-Jeffery Reactions under BallMilling Conditions Applied to the Synthesis of Unnatural Amino Acids Precursors and Indoles. Synthesis 2006, 2006, 1183-1189.

(16) Brown, A. D.; Bunnage, M. E.; Butcher, K. J.; Glossop, P. A.; James, K.; Lane, C. A. L.; Lewthwaite, R. A.; Price, D. A. Compounds Having beta-Agonist Activity. World Patent WO/2005/092841, Oct 6, 2005.

(17) Noar, J. B.; Banaszczyk, M. G.; Millan, V.; Valkirs, G.; Buechler, K. F.; Noland, B. Reactive Heterocyclic Derivatives and Methods for Their Synthesis and Use. World Patent WO/2007/075931, Jul 5, 2007.

(18) Thompson III, L. A.; Shi, J.; Zusi, C. F.; Dee, M. F.; Macor, J. E. Indole Acetic Acid Acyl Guanidines as beta-Secretase Inhibitors. U.S. Patent 7601751, Oct 13, 2009.

(19) Wardman, P.; Folkes, L. K.; Dachs, G. U.; Rossiter, S.; Greco, O. Use of Indole-3-Acetic Acid Derivatives in Medicine. World Patent WO/2002/002110, Jan 10, 2002. 


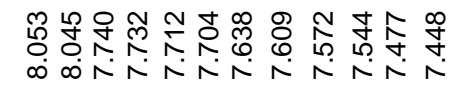

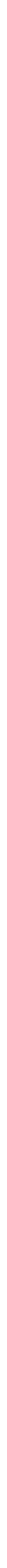




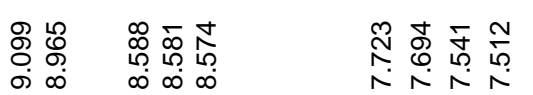
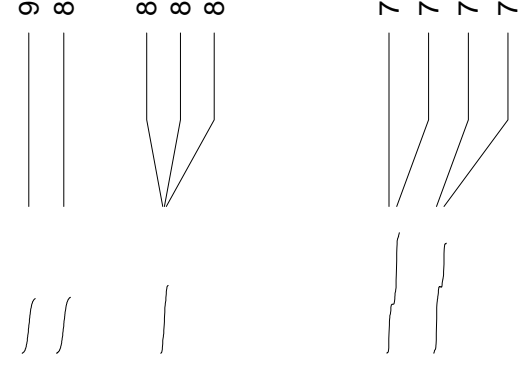

$\mathrm{H}_{2} \mathrm{O}$

$\mathrm{CD}_{3} \mathrm{OD}$ 

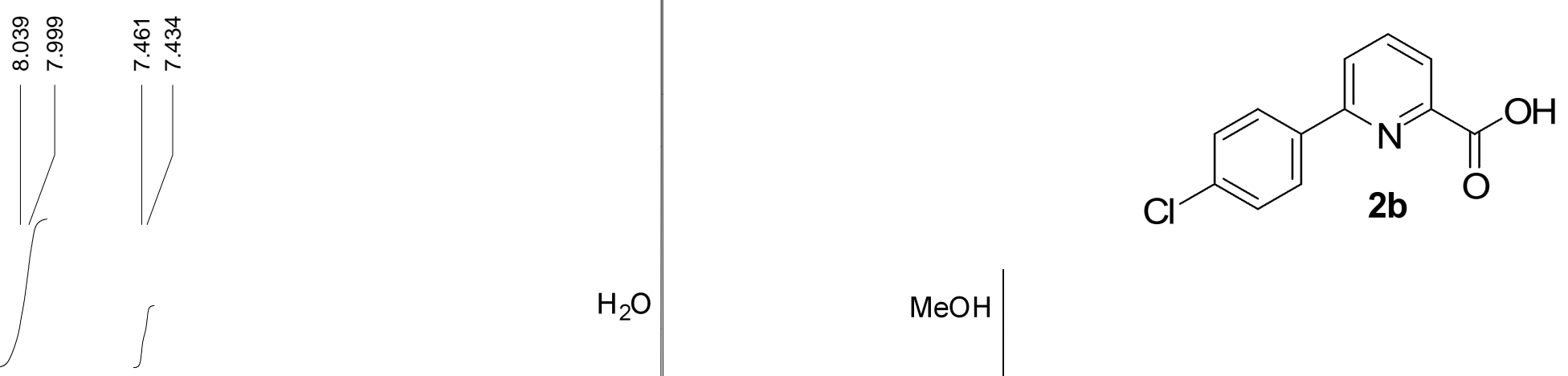

$\mathrm{CD}_{3} \mathrm{OD}$

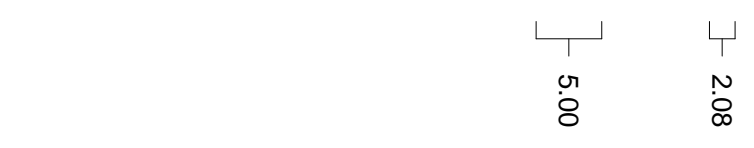

$$
10.0
$$

9.0

8.0

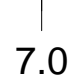

6.0

4.0

3.0

2.0

1.0

S14 0.0

ppm (t1) 

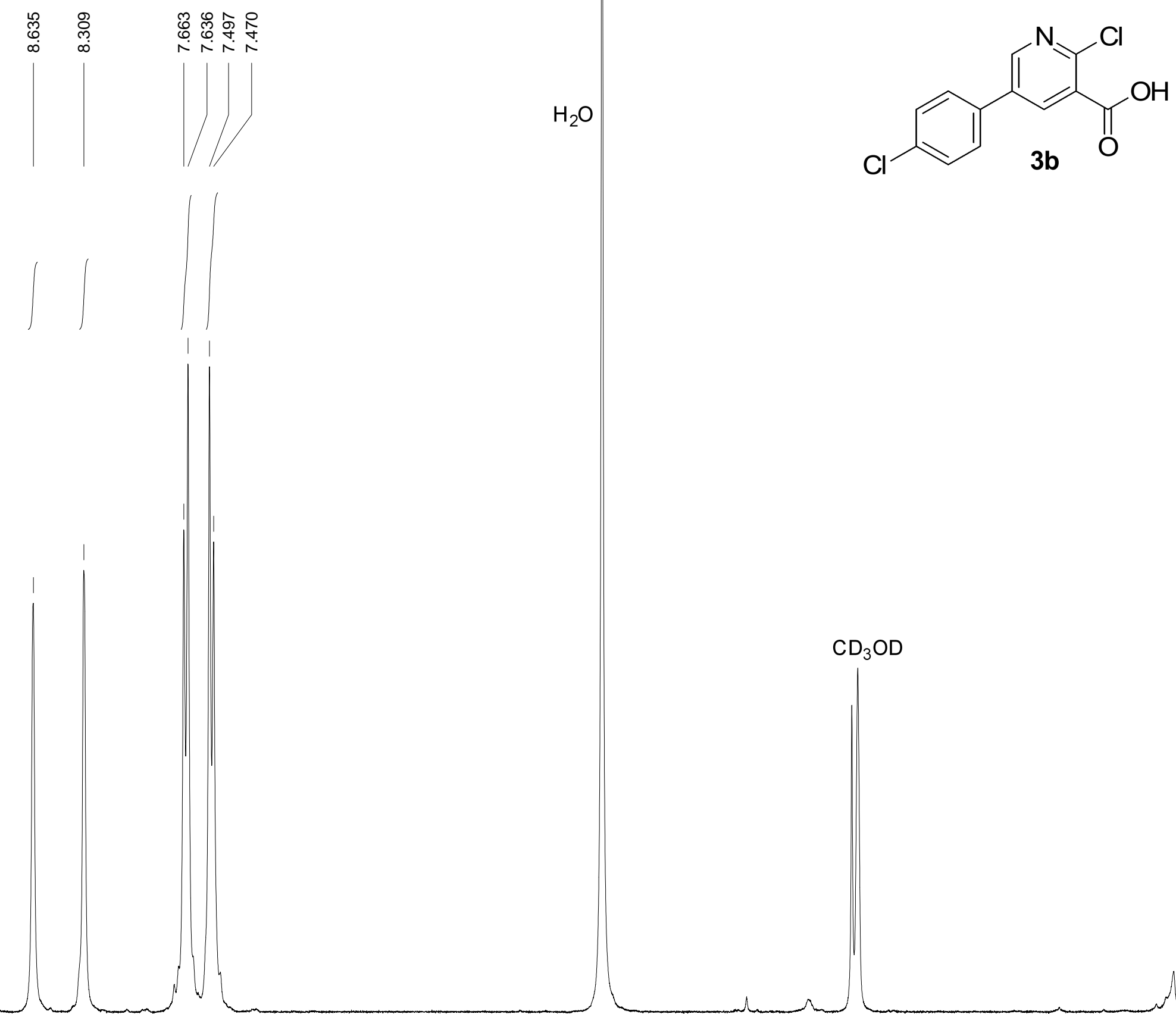

$\mathrm{CD}_{3} \mathrm{OD}$

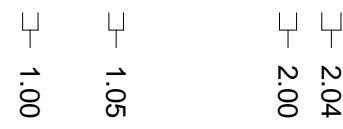

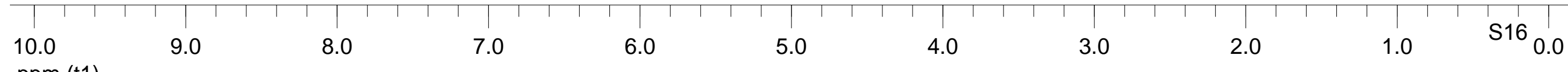




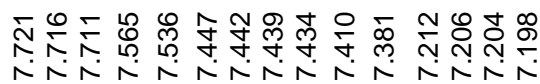
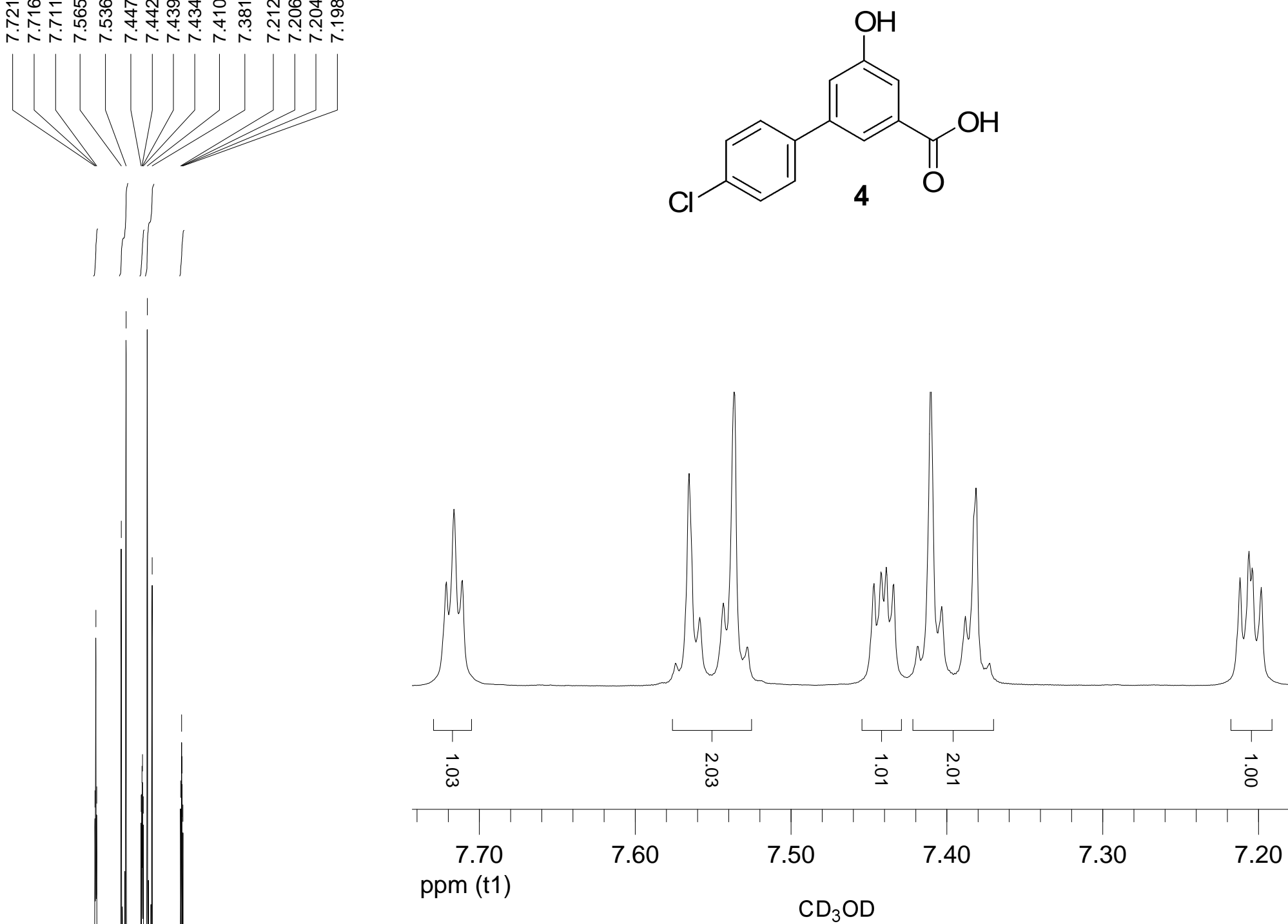

㝘总兽

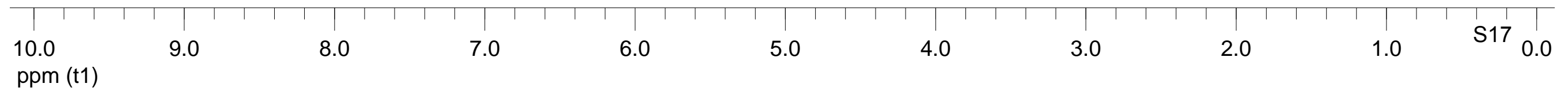




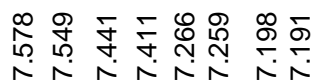

$\hat{\imath} \hat{\imath} \hat{\imath} \hat{i} \hat{i}$

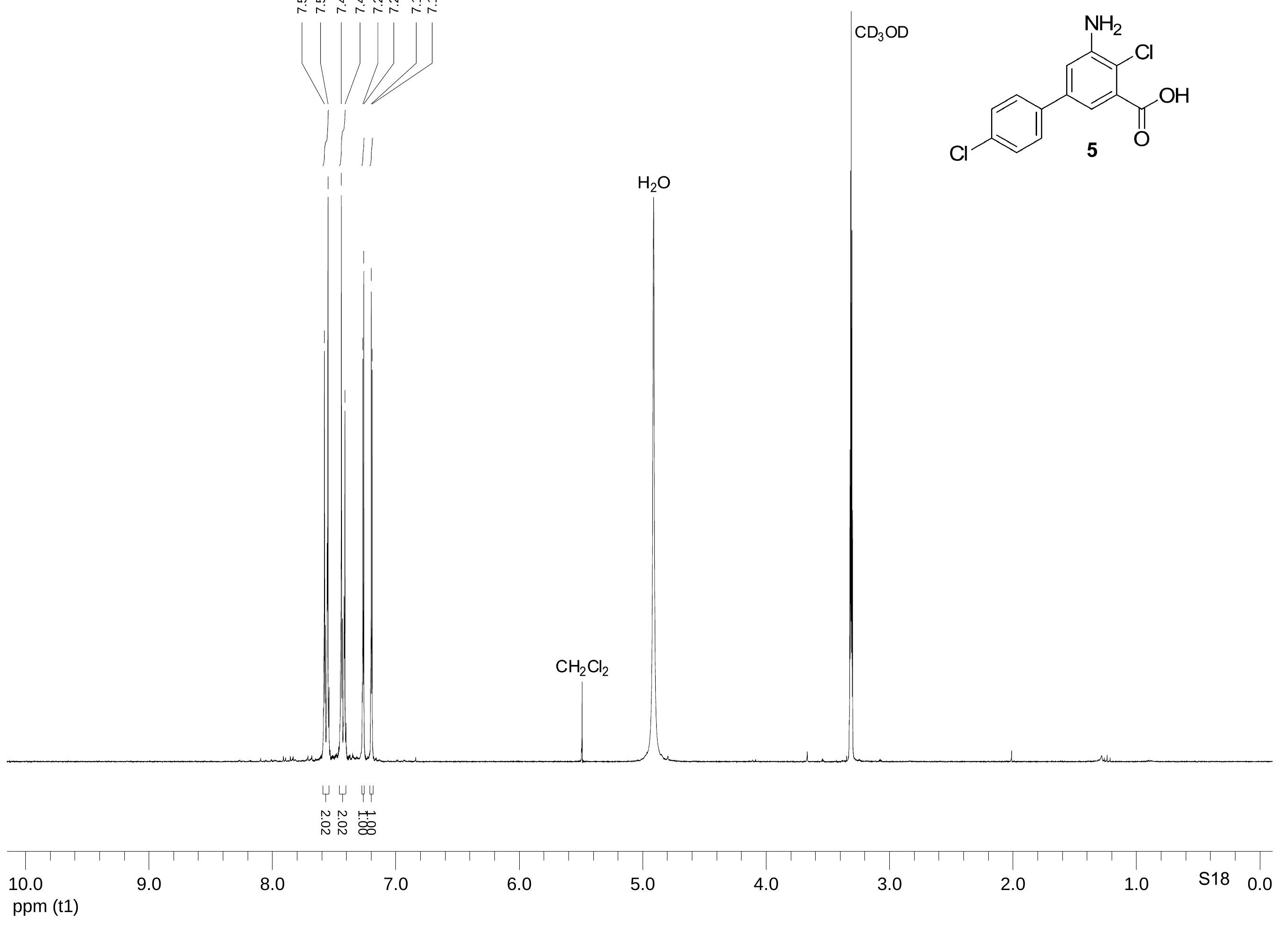




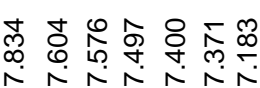
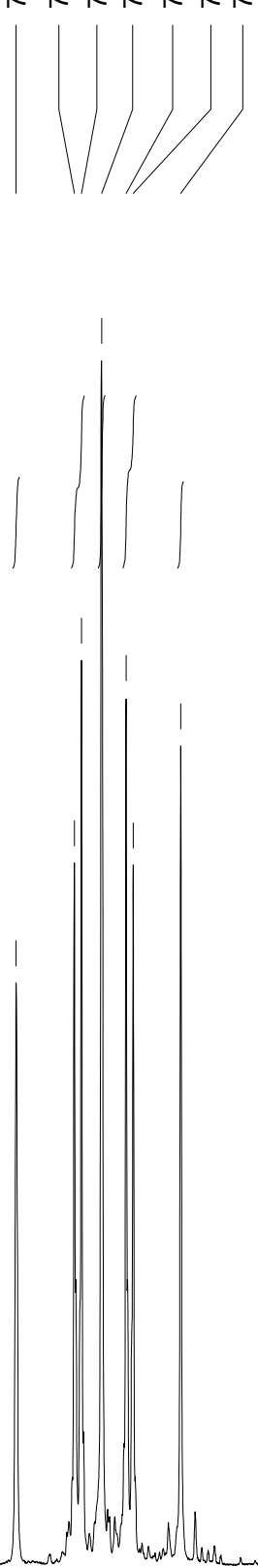

$\biguplus \quad \biguplus \biguplus \quad$

ํำ
$\mathrm{H}_{2} \mathrm{O}$

$\mathrm{CD}_{3} \mathrm{OD}$

$\mathrm{MeOH}$
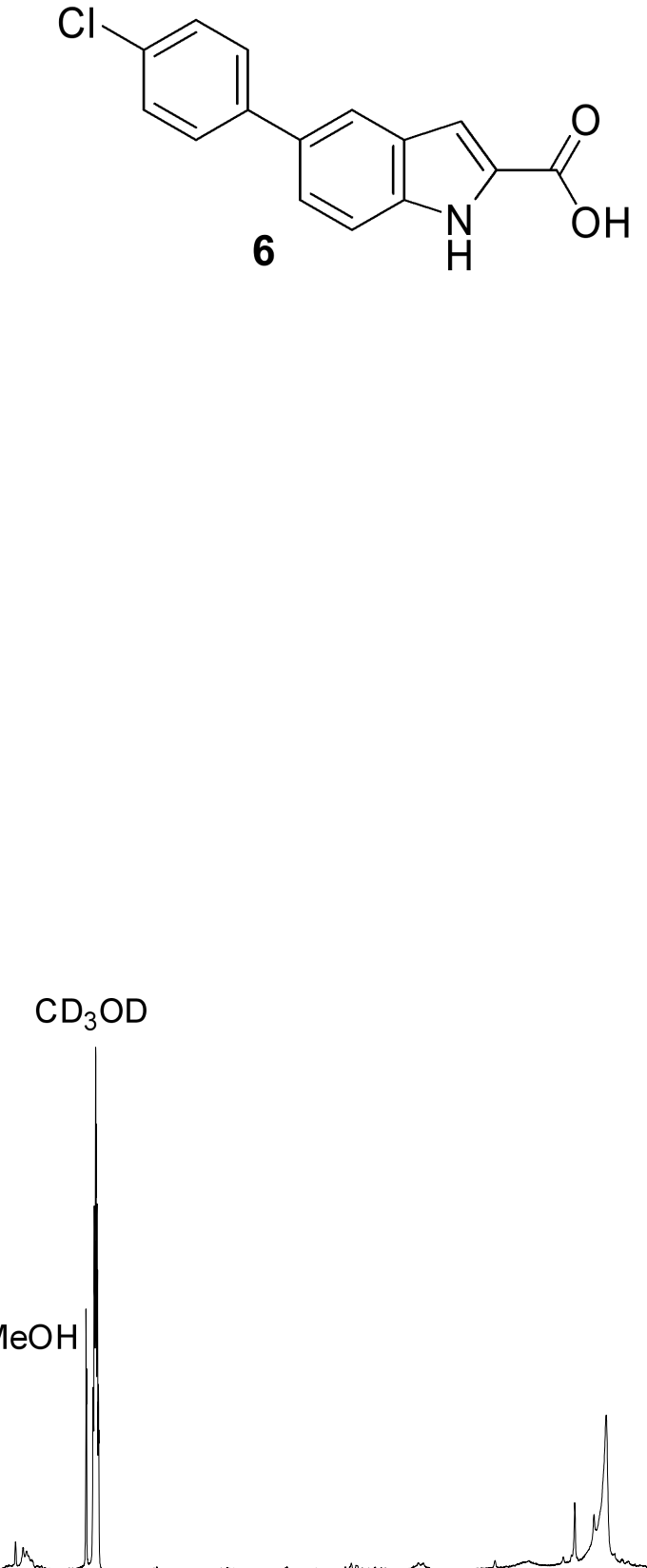

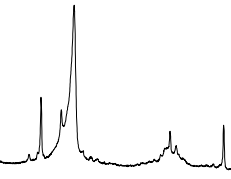

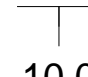

10.0

ppm (t1) 


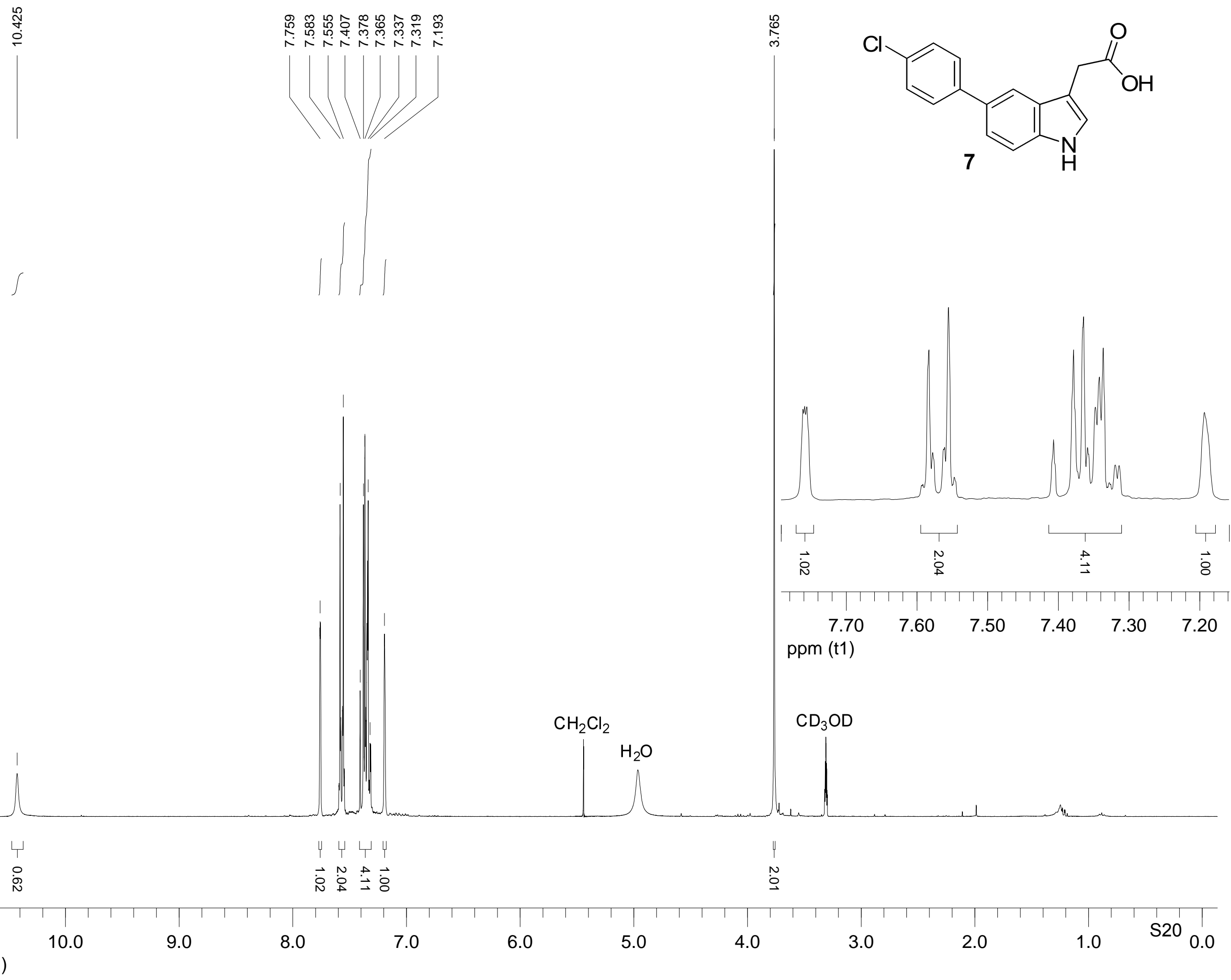



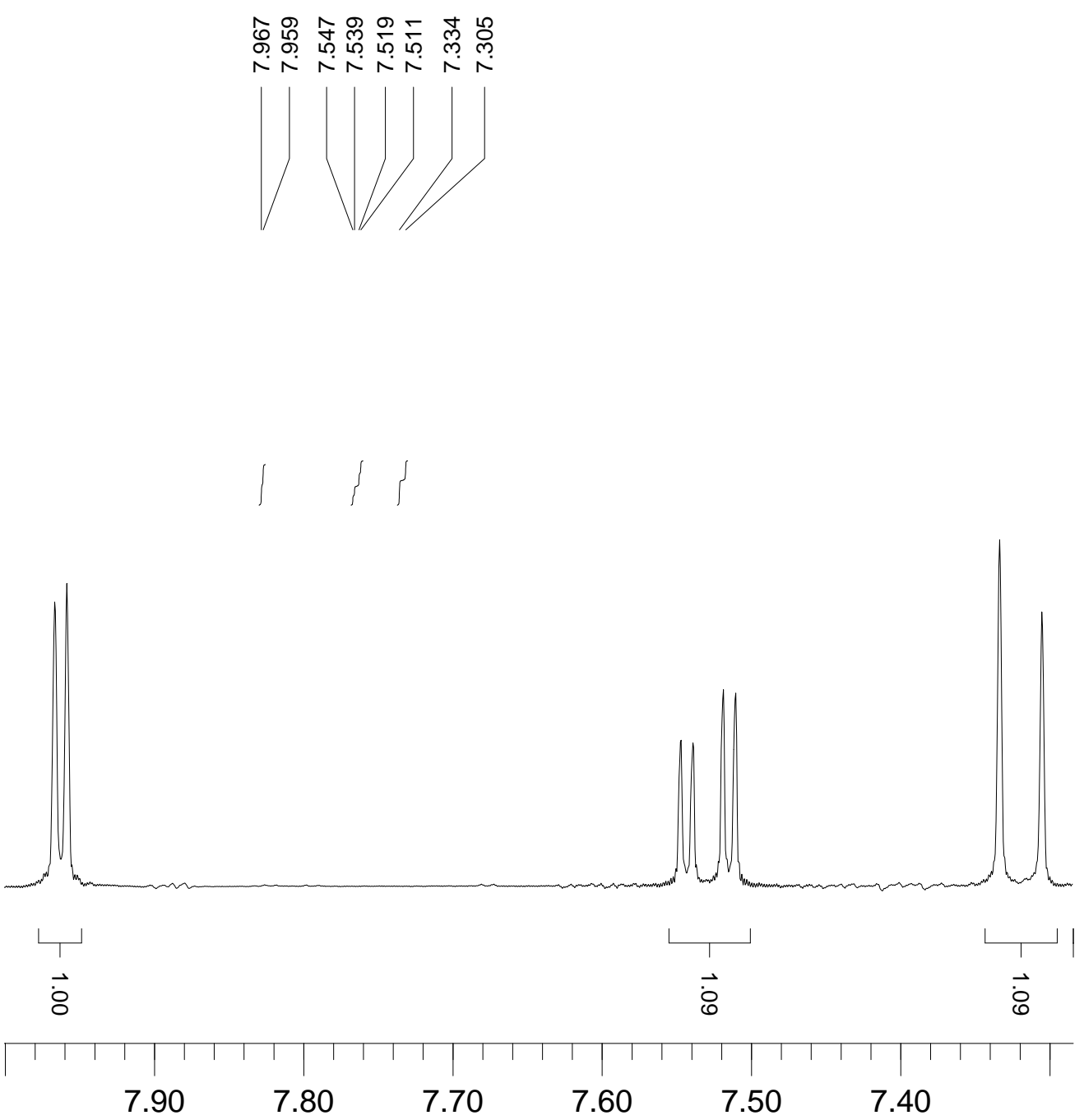

ppm (t1)

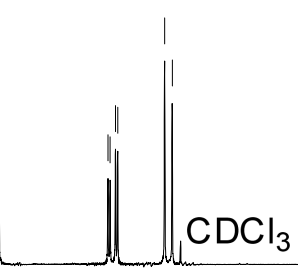

$$
\begin{array}{r}
\psi \\
\hline
\end{array}
$$

若占客

$\underset{b}{\omega}$ 


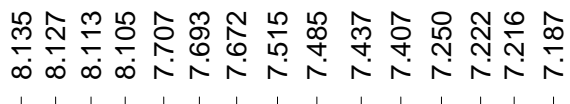

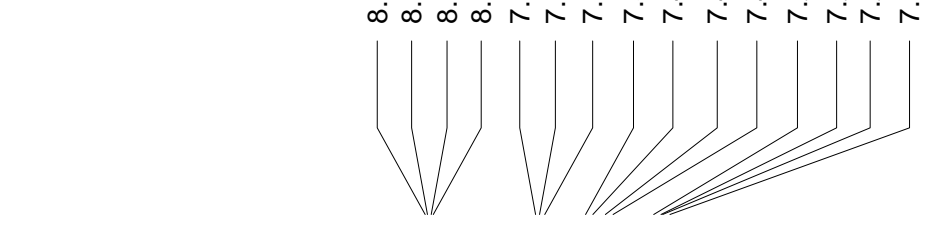<smiles>COC(=O)c1cc(-c2ccc(Cl)cc2)ccc1F</smiles>

$1 / 1\}$

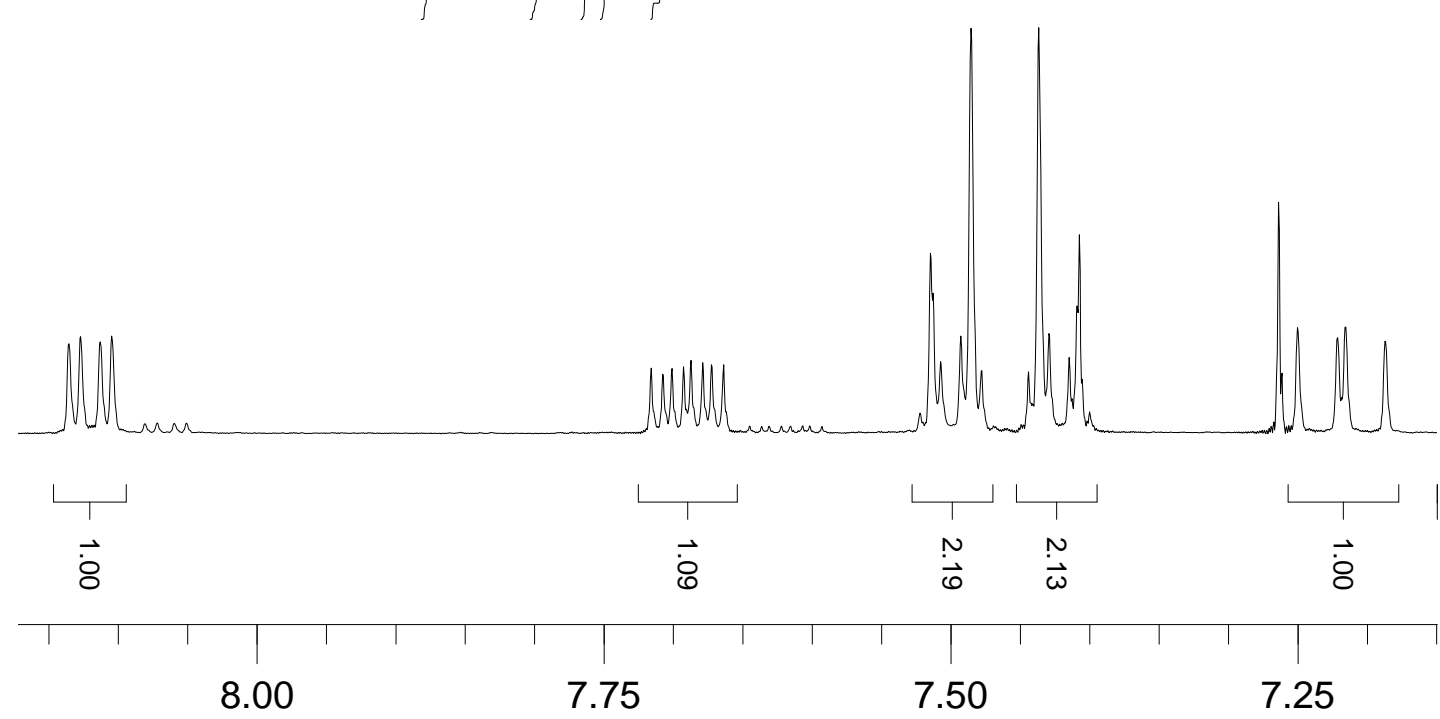

ppm (t1)

75

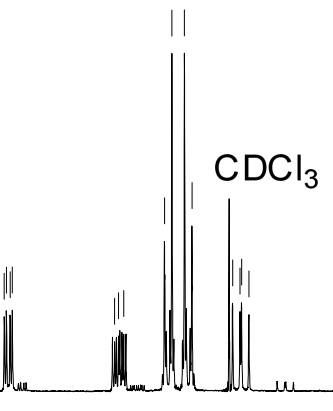

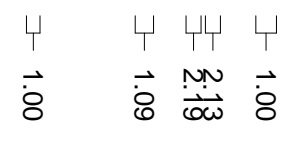

4
$\omega$
$\vdots$
$\vdots$
$\vdots$

$\mathrm{H}_{2} \mathrm{O}$ 

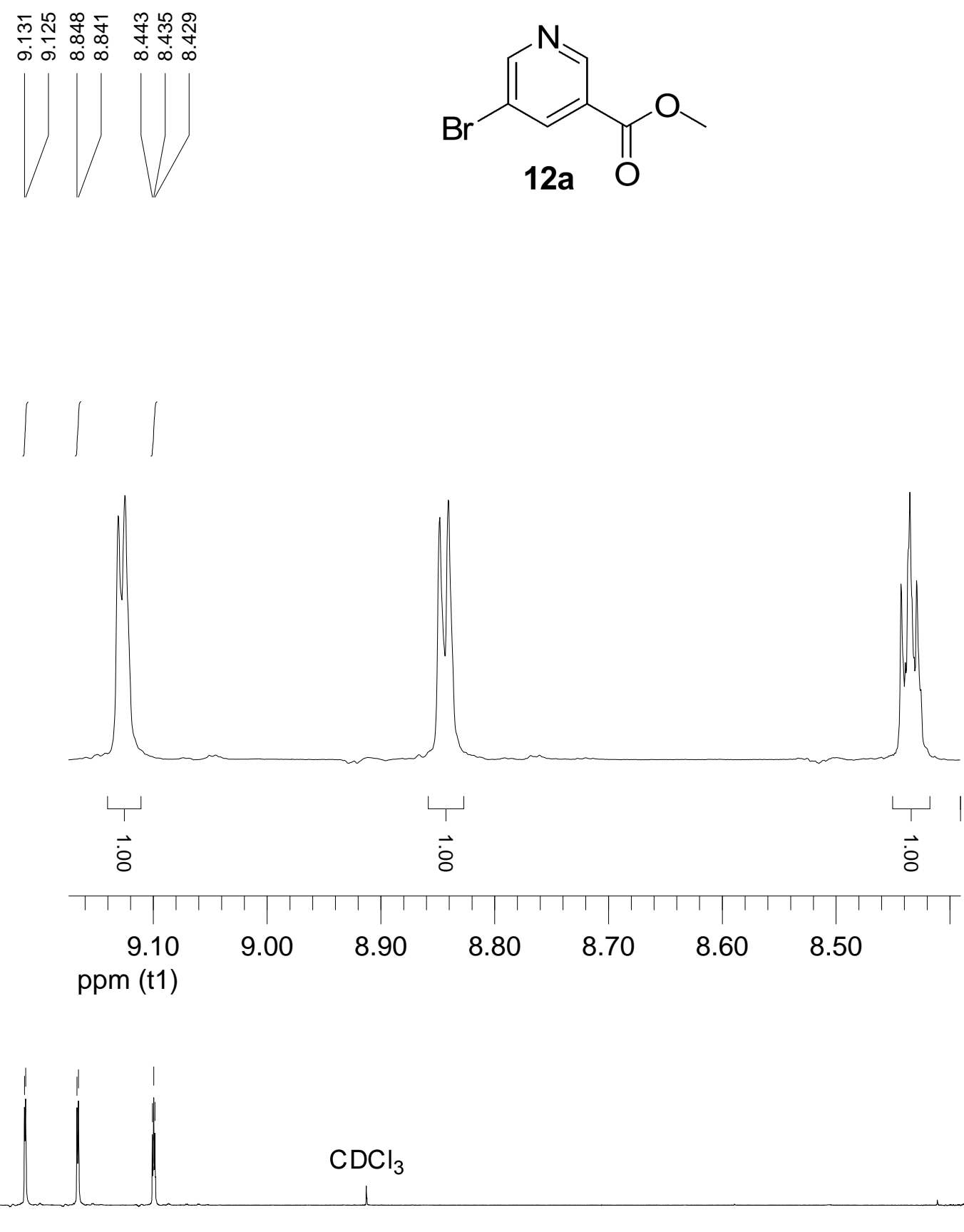

$\mathrm{CDCl}_{3}$

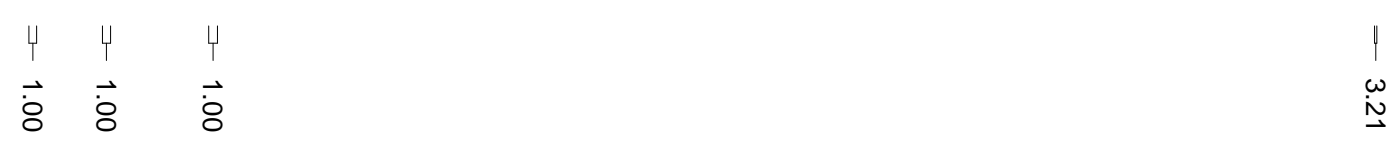




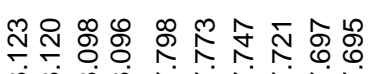

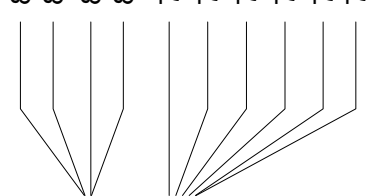

8

ppm (t1)

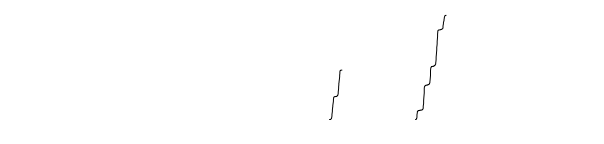

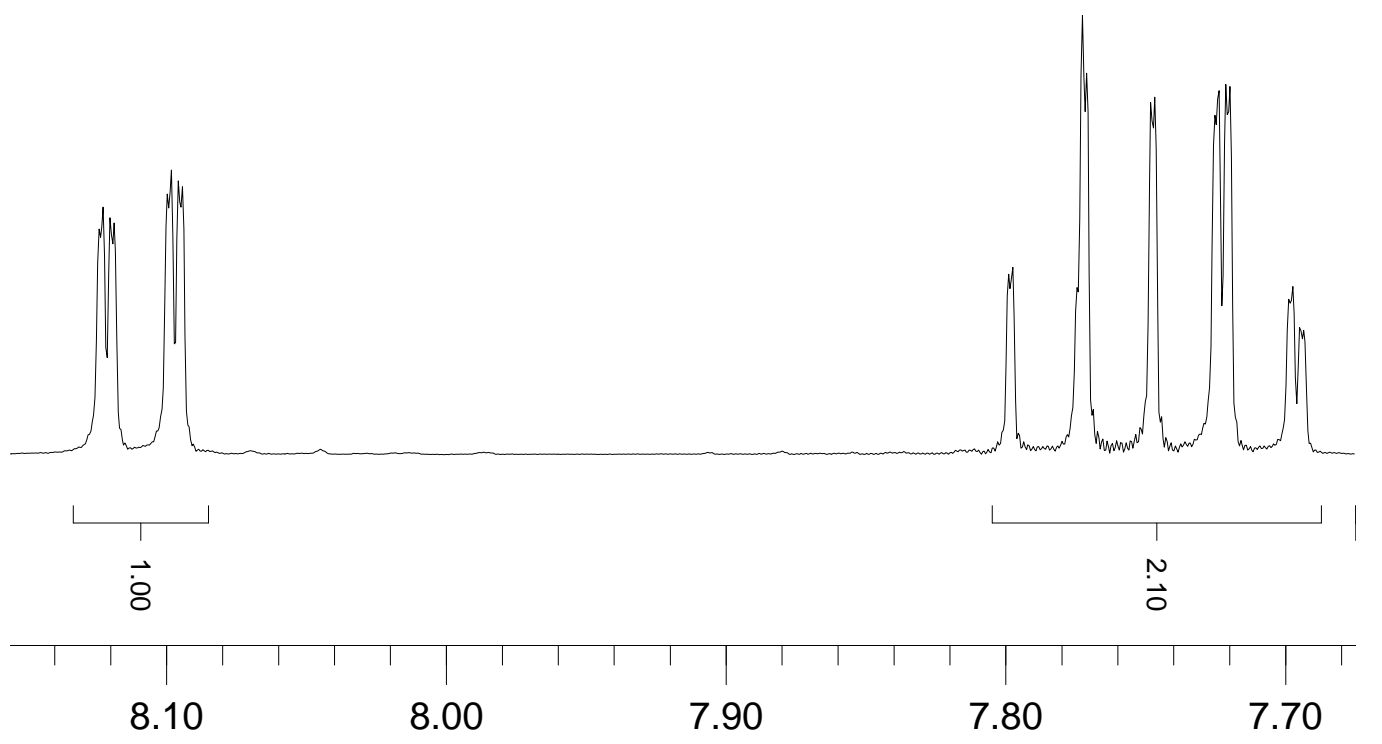

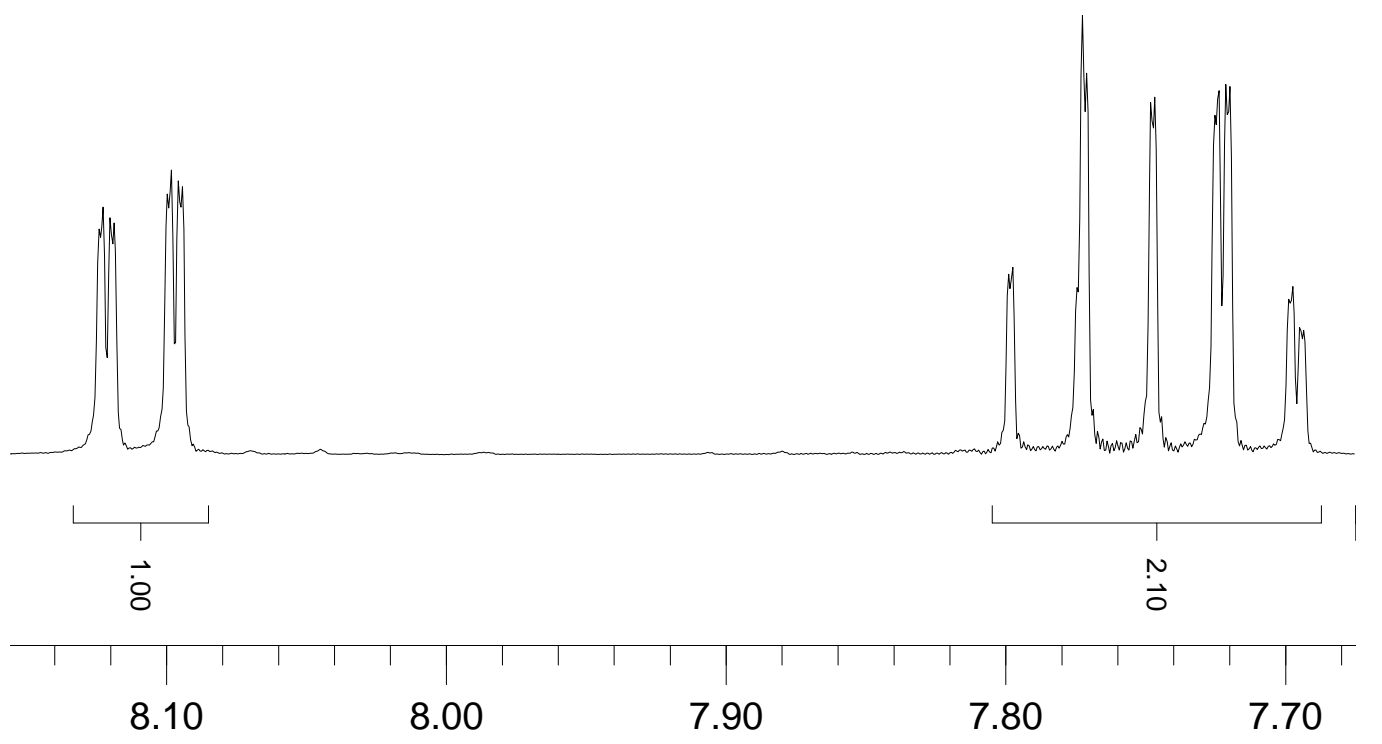

$\stackrel{\infty}{\stackrel{\infty}{+}}$<smiles>COC(=O)c1cccc(Br)n1</smiles>

$2 b$

$\mathrm{CDCl}_{3}$

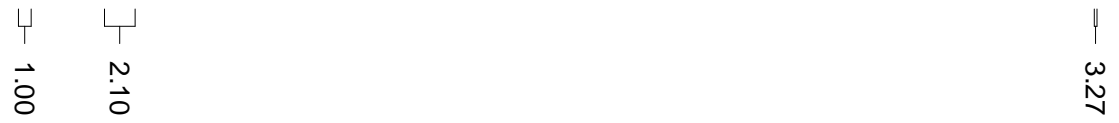

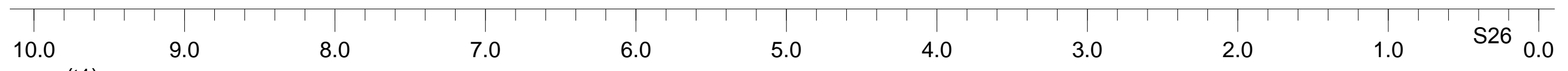

ppm (t1) 


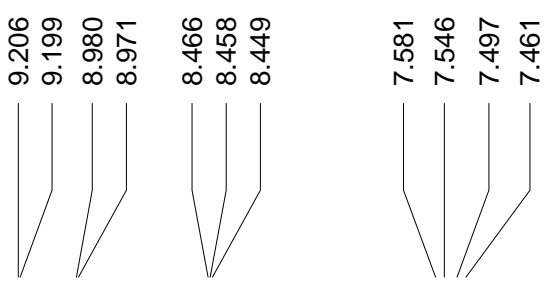<smiles>COC(=O)c1cncc(-c2ccc(Cl)cc2)c1</smiles>

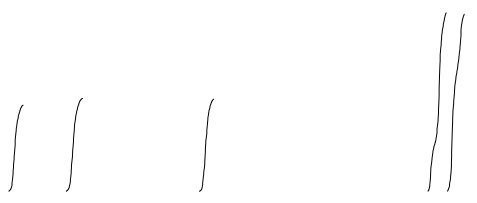

$\mathrm{CDCl}_{3}$
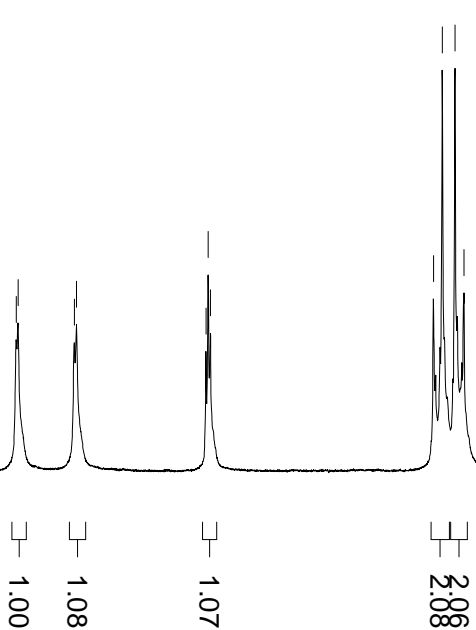

$\stackrel{1}{8}$

9

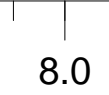

$\infty$

ppm (t1)

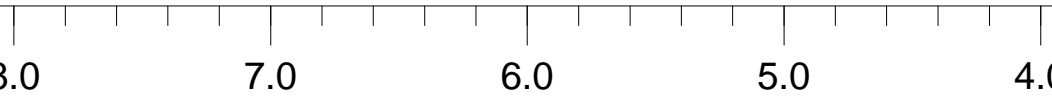

\begin{tabular}{ll|l|l|}
$\mid$ & & & $\mid$ \\
4.0 & & 3.0
\end{tabular}

2.0

1.0

S27. 0 


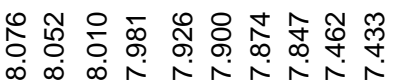

|||||||| $\mid$
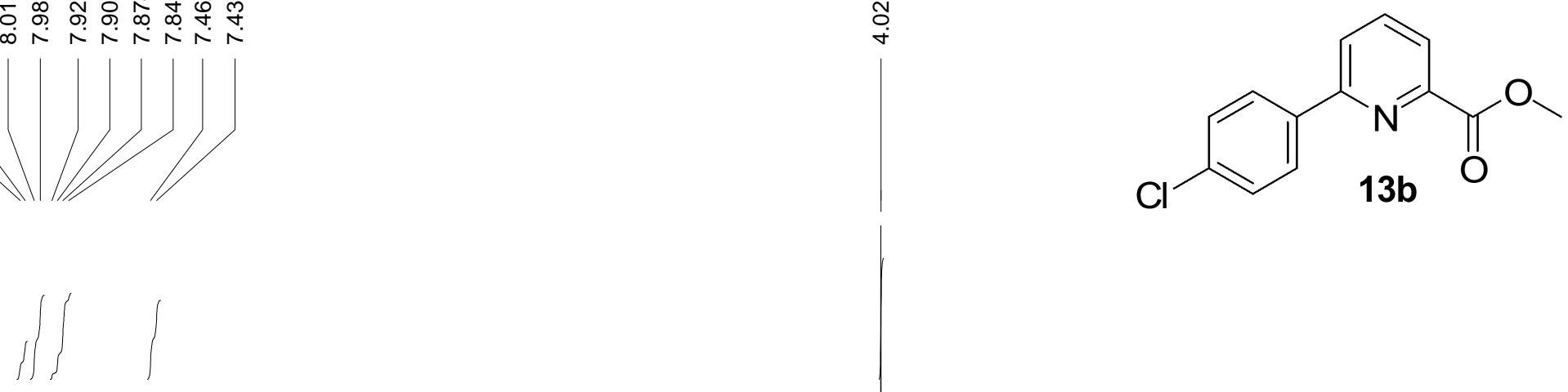

$\mathrm{CDCl}_{3}$

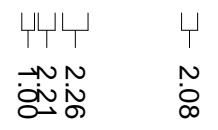

4
$\omega$
$\vdots$
$\vdots$
0

8.0

3.0

2.0

1.0

$\mathrm{S}^{\mathrm{S}}{ }_{0.0}$ 

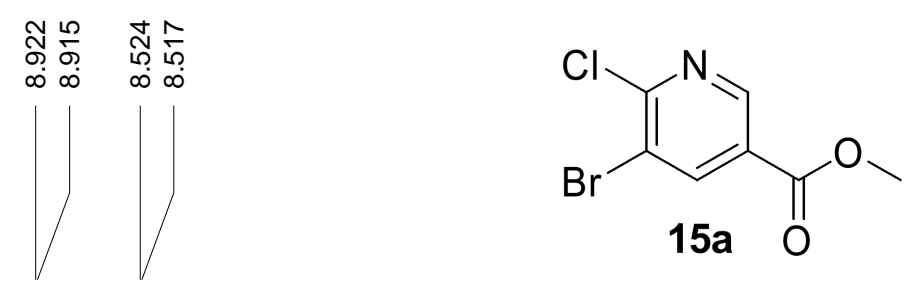

$\mathrm{CDCl}_{3}$

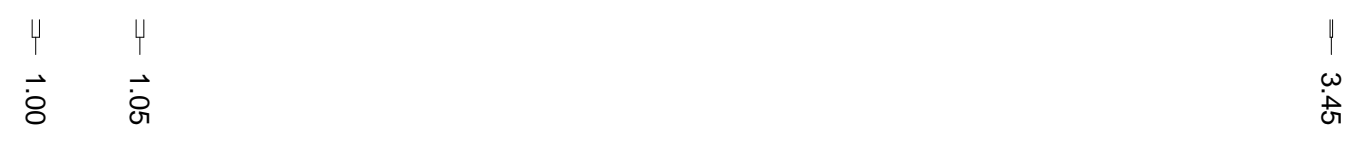

$$
10.0
$$

ppm (t1)

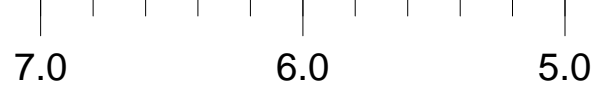

3.0

2.0

1.0

S29 0.0 

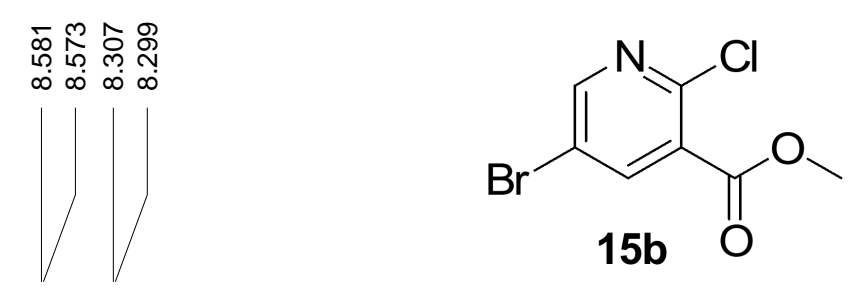

$15 b \mathrm{O}$

$\mathrm{CDCl}_{3}$

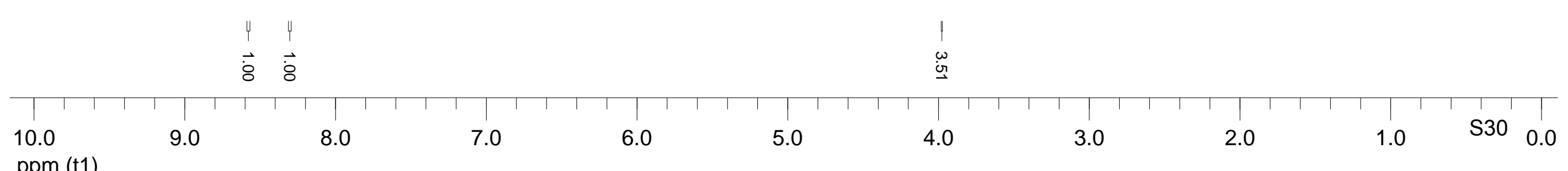



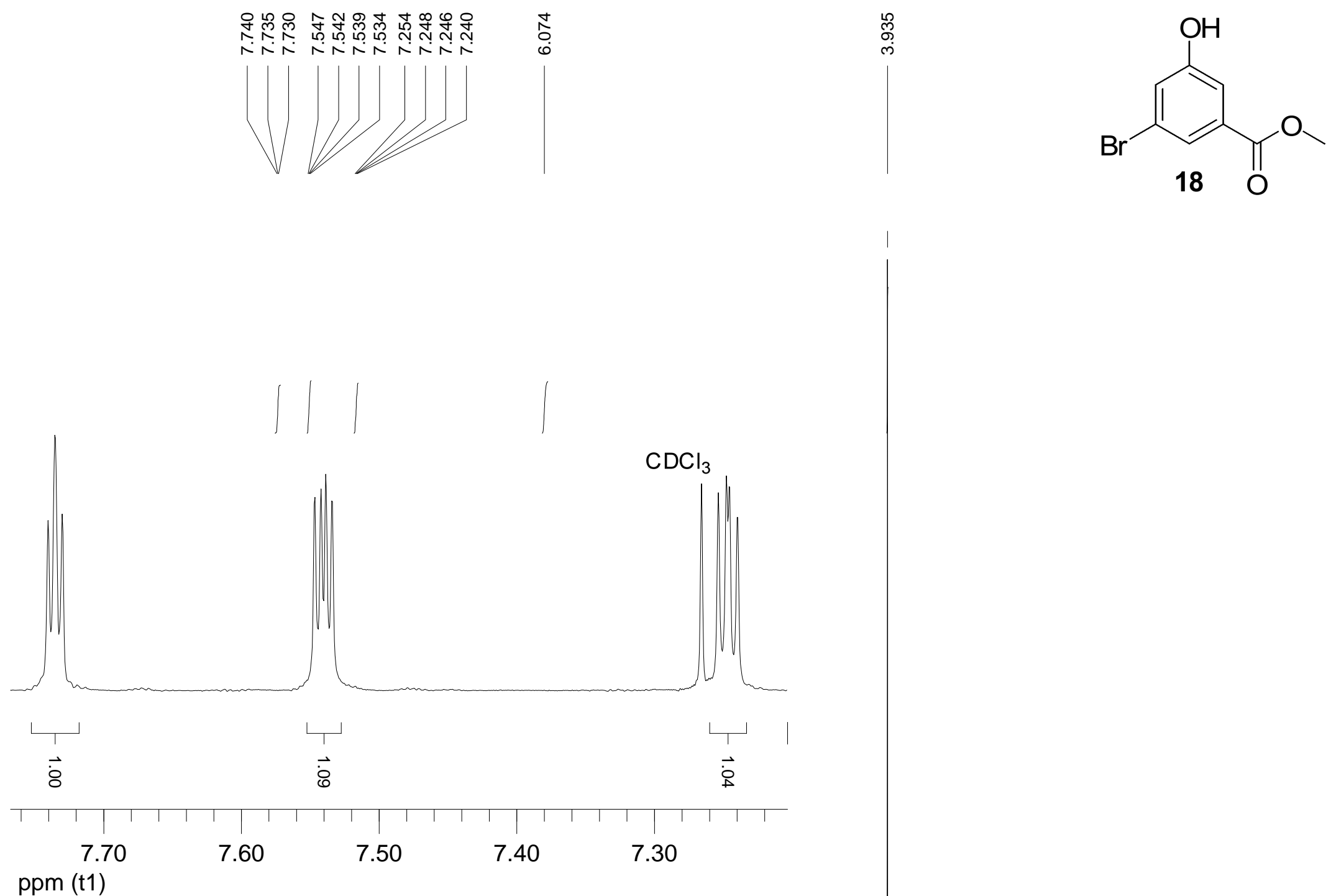

ppm (t1)

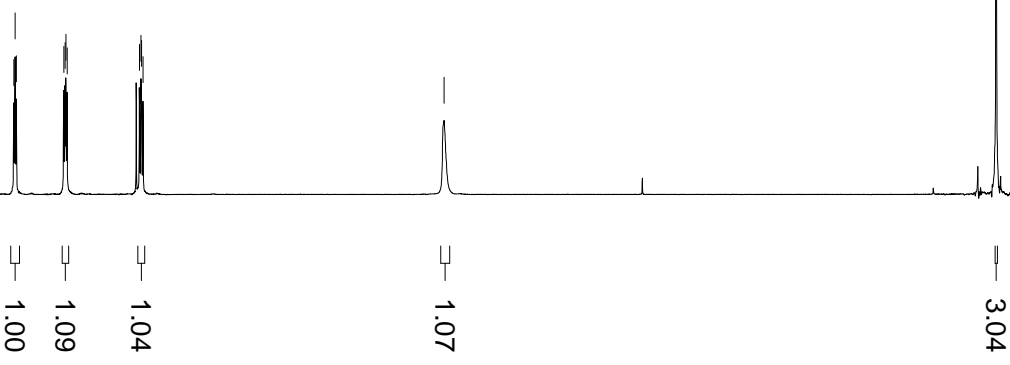

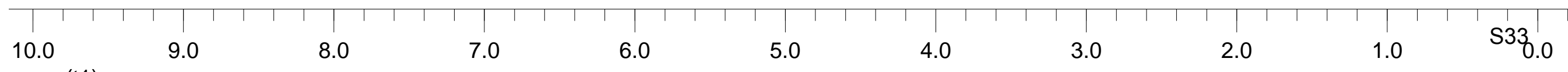



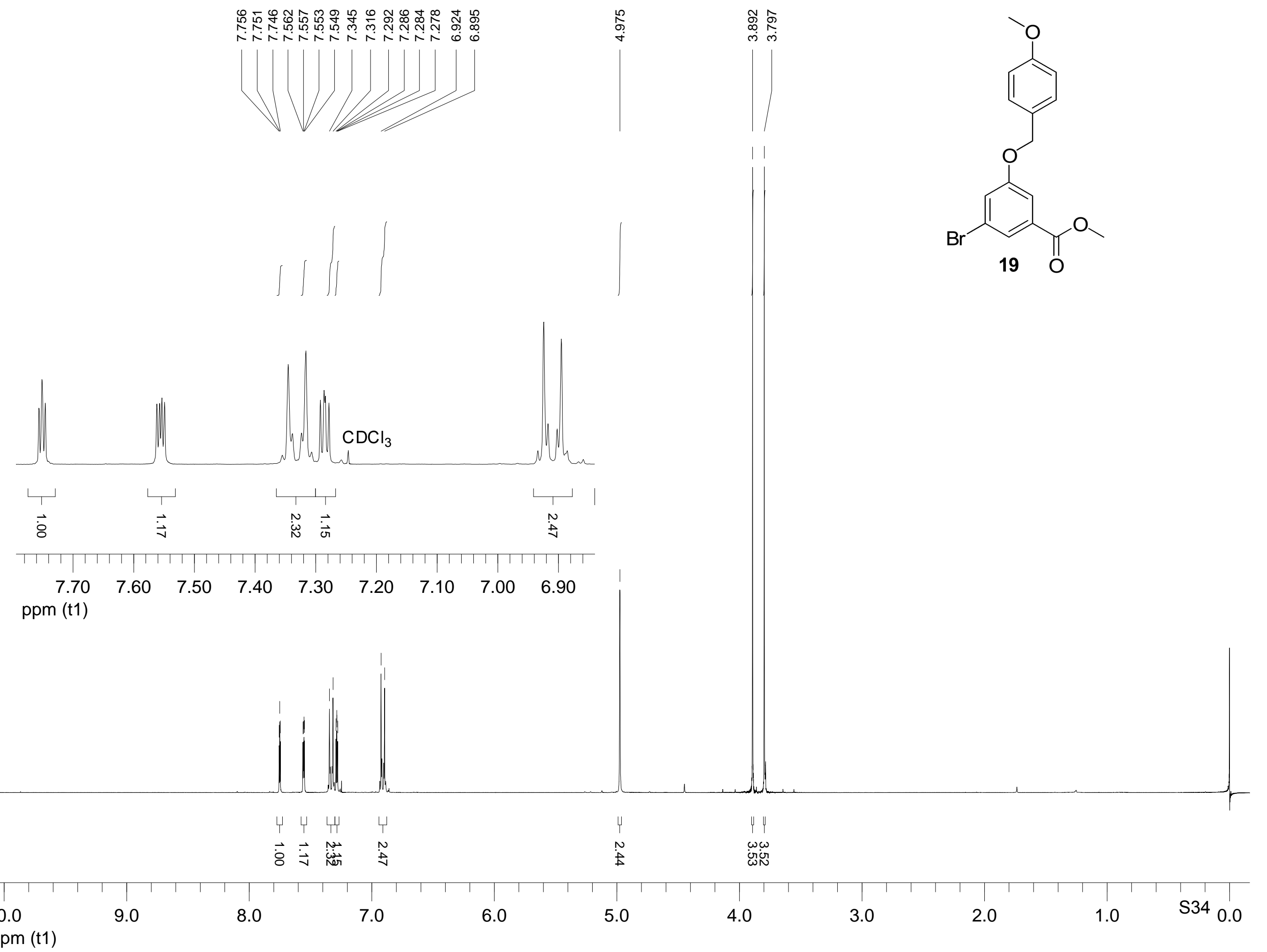

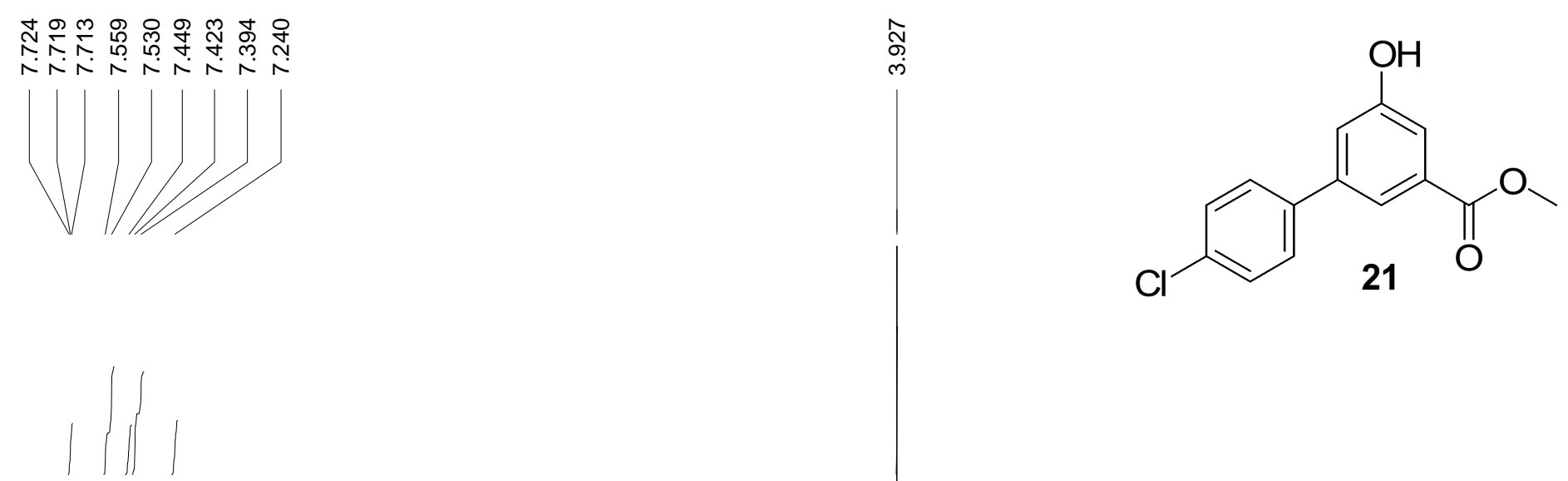

\section{$\mathrm{CD}_{3} \mathrm{OD}$}

४

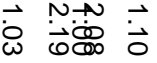

\&̊ 


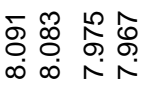

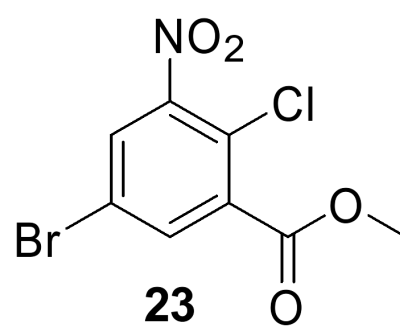

$\mathrm{CDCl}_{3}$

보용 

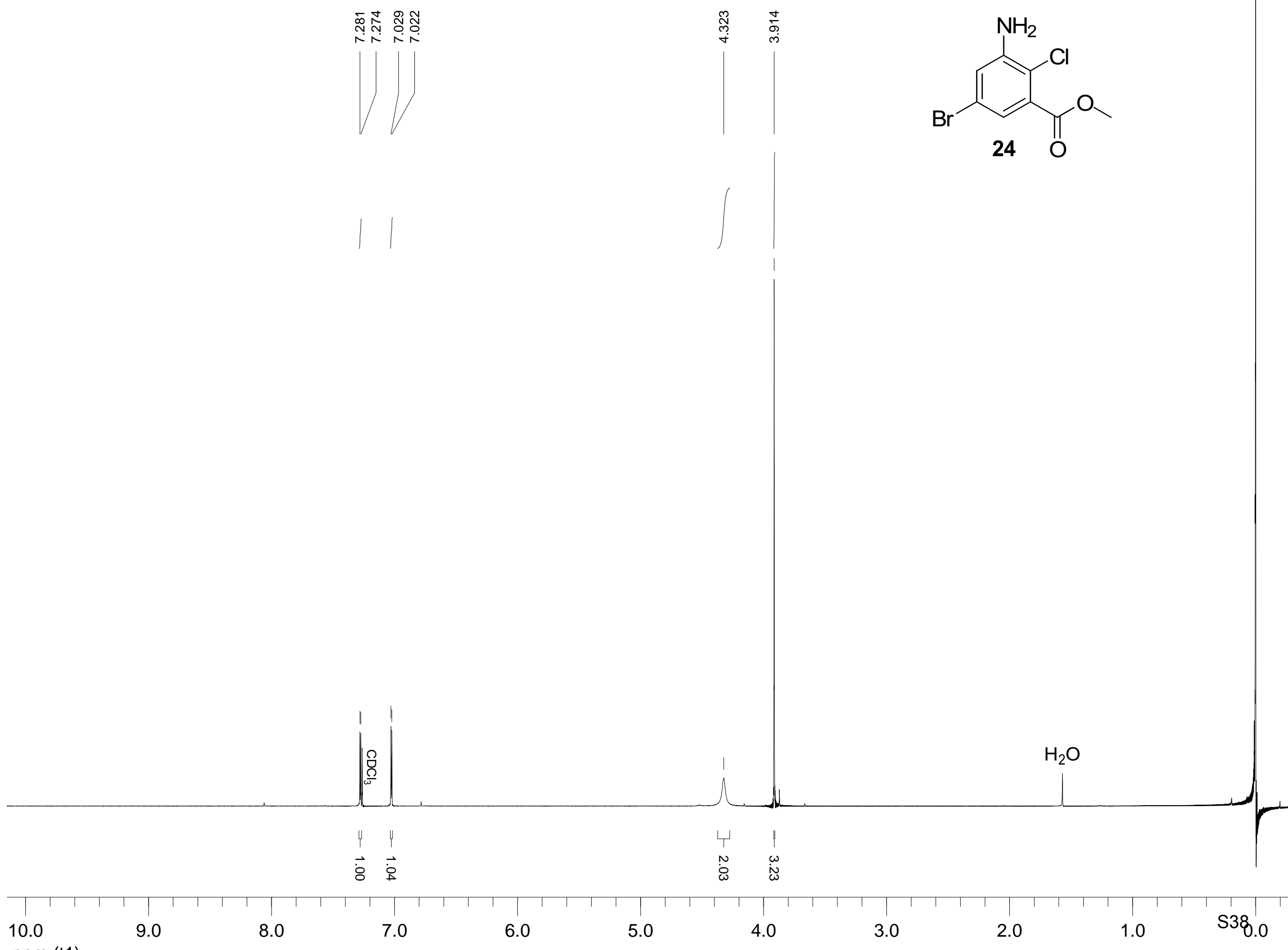

$\mathrm{H}_{2} \mathrm{O}$ 


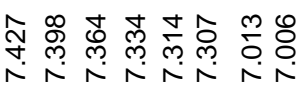
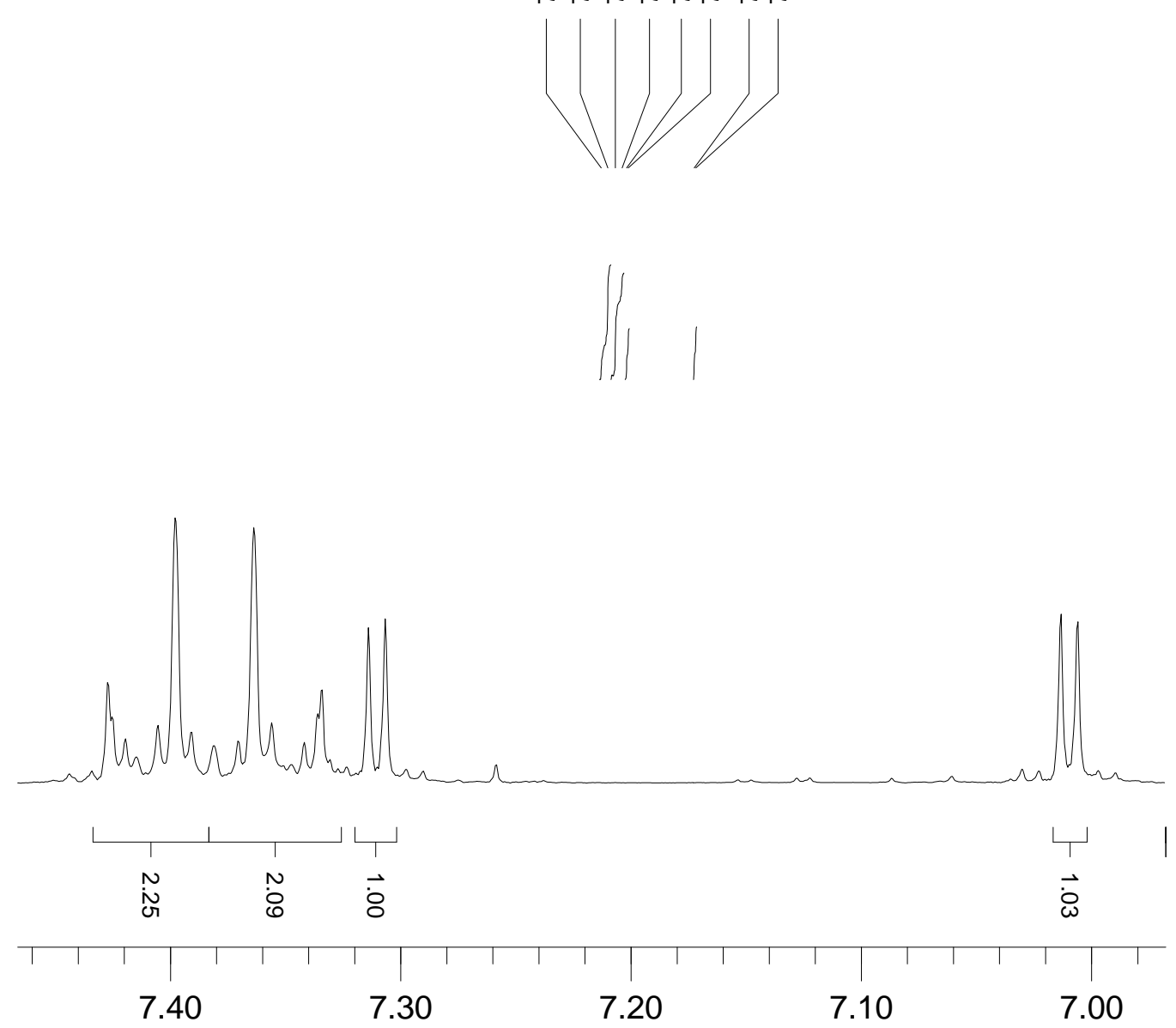

ppm (t1)
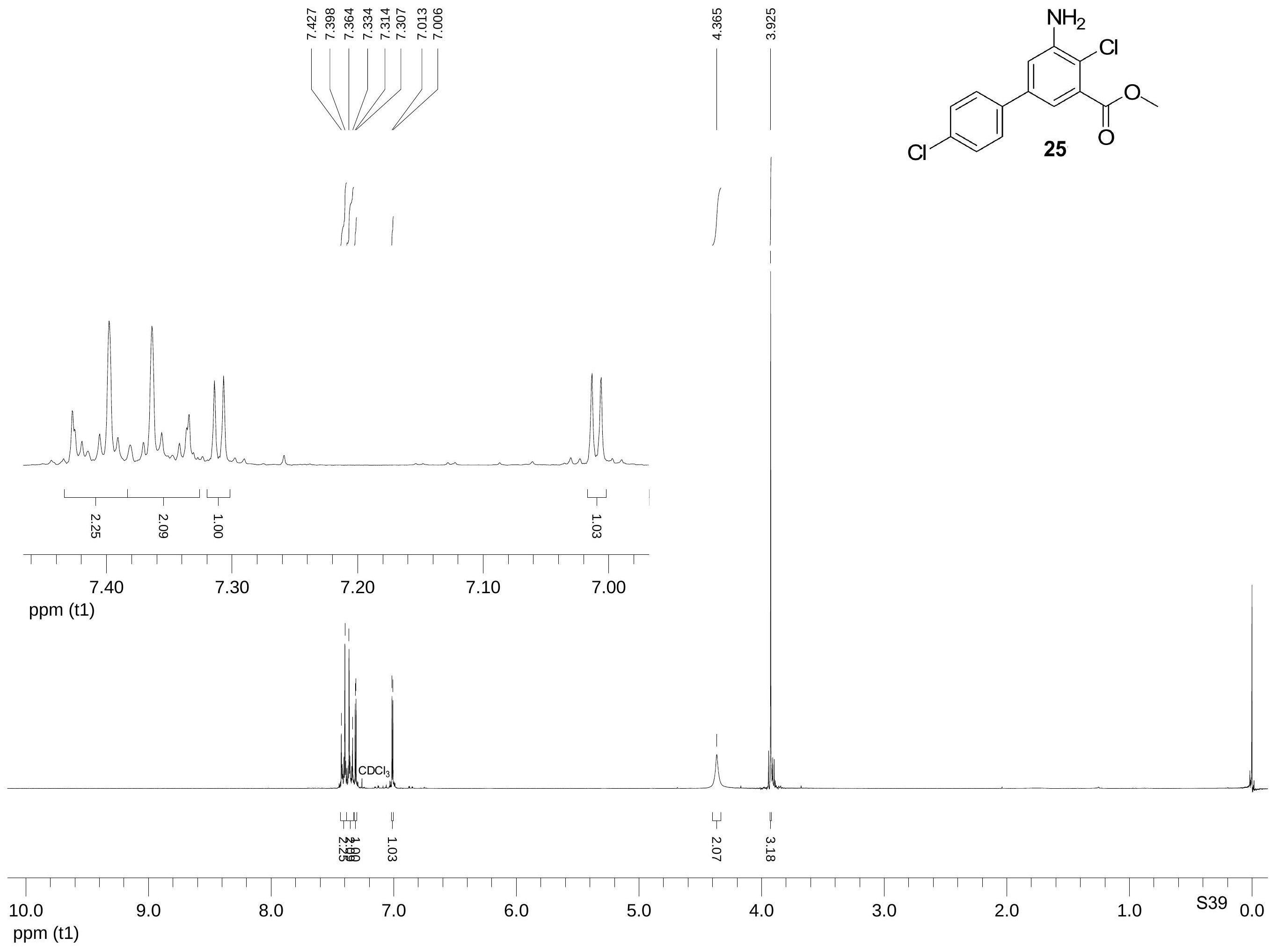


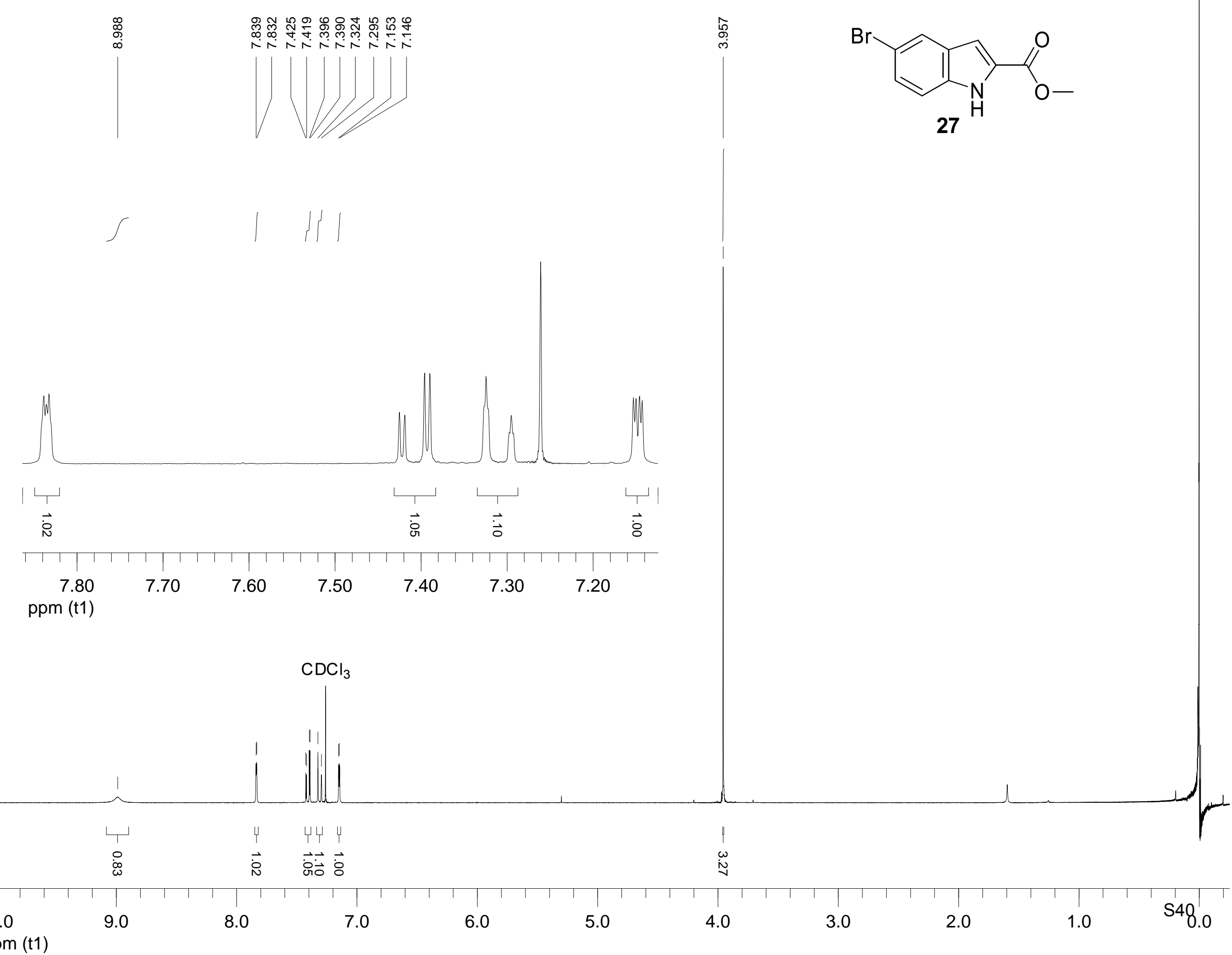


蛋

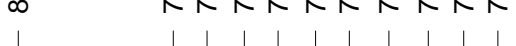

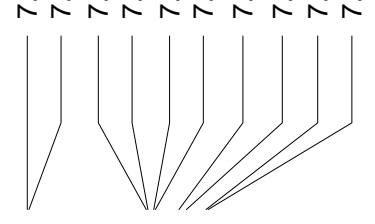

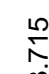<smiles>COC(=O)Cc1c[nH]c2ccc(Br)cc12</smiles>

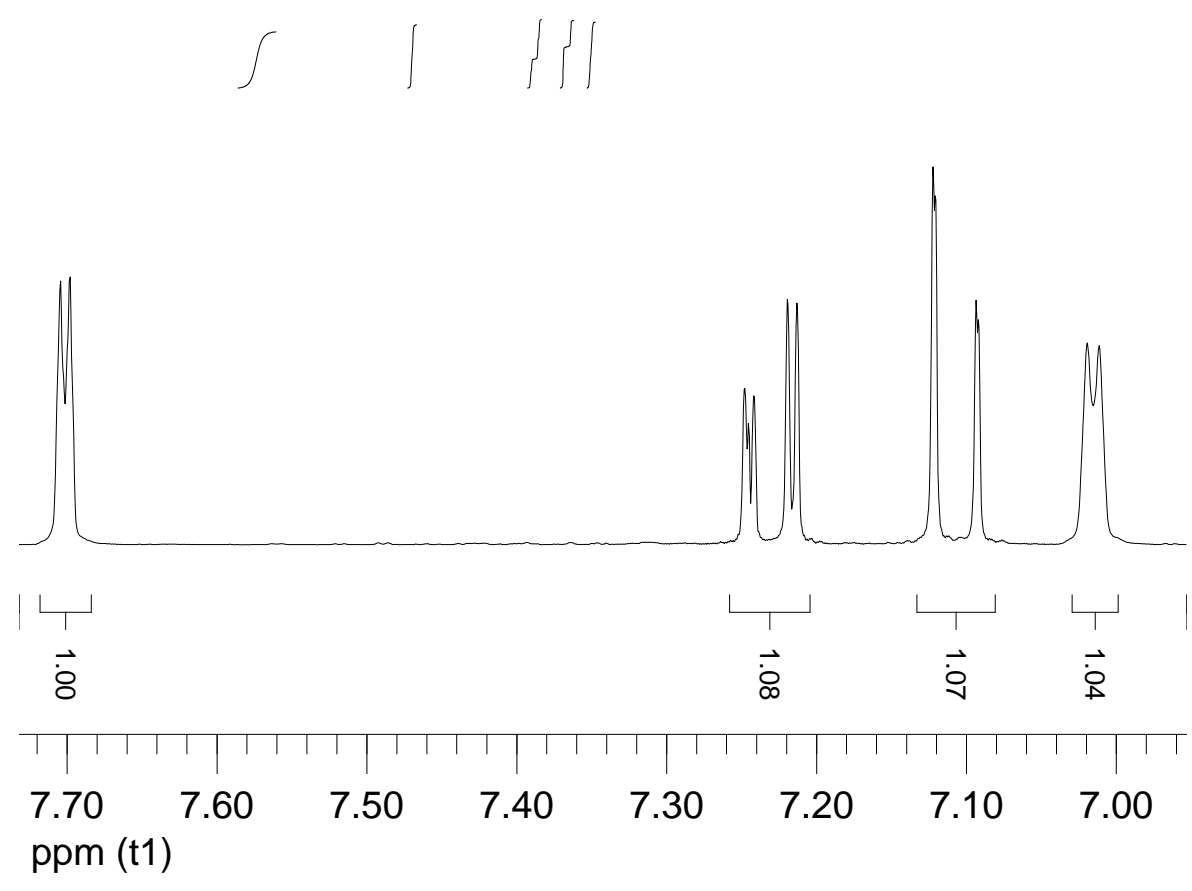

ppm (t1)
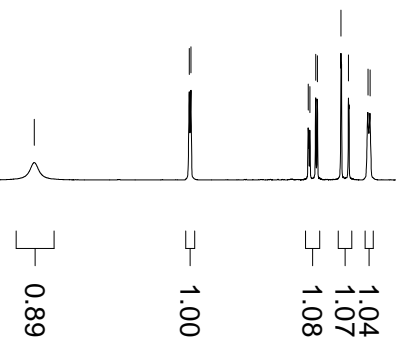

$\dot{\leftrightarrow}$

1

ppm (t1) 
索

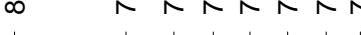
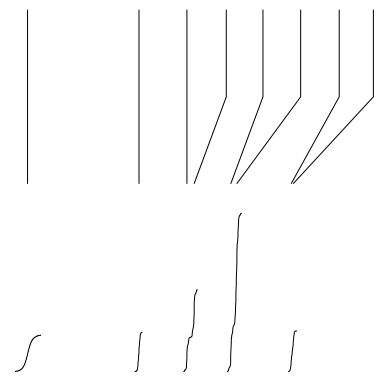

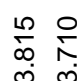

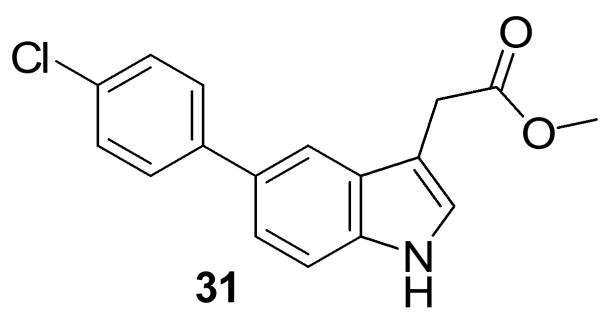

EtOAc

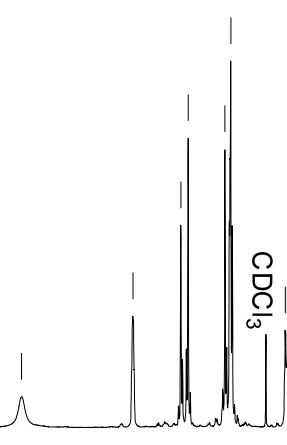

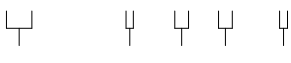

总

$\stackrel{\circ}{n \infty}$

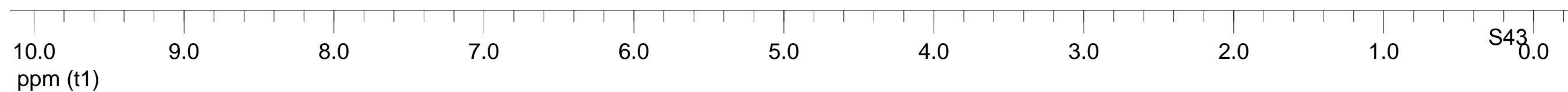

